\title{
Beilinson-Drinfeld Schubert varieties and global Demazure modules
}

\author{
Ilya Dumanski ${ }^{1,2}$, Evgeny Feigin ${ }^{3,4}$ and Michael Finkelberg 5,6,7 \\ ${ }^{1}$ Department of Mathematics, HSE University, Usacheva str. 6, Moscow, 119048, Russia. \\ ${ }^{2}$ Independent University of Moscow, Bolshoy Vlasyevskiy Pereulok 11, Moscow, 119002, Russia; \\ E-mail: ilyadumnsk@gmail.com. \\ ${ }^{3}$ Department of Mathematics, HSE University, Usacheva str. 6, Moscow, 119048, Russia. \\ ${ }^{4}$ Skolkovo Institute of Science and Technology, Skolkovo Innovation Center, Building 3, Moscow, 143026, Russia; \\ E-mail: evgfeig@gmail.com. \\ ${ }^{5}$ Department of Mathematics, HSE University, Russia, Usacheva str. 6, Moscow, 119048, Russia. \\ ${ }^{6}$ Skolkovo Institute of Science and Technology, Skolkovo Innovation Center, Building 3, Moscow, 143026, Russia. \\ ${ }^{7}$ Institute for Information Transmission Problems of RAS, Moscow, Russia; E-mail: fnklberg@gmail.com.
}

Received: 30 May 2020; Revised: 11 March 2021; Accepted: 24 April 2021

2020 Mathematics Subject Classification: Primary - 14M15; Secondary - 17B67

\begin{abstract}
We compute the spaces of sections of powers of the determinant line bundle on the spherical Schubert subvarieties of the Beilinson-Drinfeld affine Grassmannians. The answer is given in terms of global Demazure modules over the current Lie algebra.
\end{abstract}

\section{Introduction}

Let $\mathfrak{g}$ be a simple complex Lie algebra. To simplify the notation, in the introduction we assume that $\mathfrak{g}$ is simply laced. We drop this restriction in the main body of the paper.

The central objects of the algebraic representation theory of $\mathfrak{g}$ are finite-dimensional irreducible representations $V_{\lambda}$ of $\mathfrak{g}$ labelled by the dominant integral weights $\lambda \in P_{+}$. The geometric objects responsible for these representations are the flag varieties. In particular, flag varieties are naturally embedded into projectivisations of irreducible $\mathfrak{g}$-modules, and the celebrated Borel-Weil theorem states that finite-dimensional $g$ modules are realised as (dual) spaces of sections of line bundles on flag varieties (see, e.g., [Fu, Kum2]). These properties are still valid after passing to the Demazure submodules inside $V_{\lambda}$ and to the Schubert subvarieties in flag varieties.

In this paper we are interested in the representation theory (algebraic and geometric) of two natural infinite-dimensional analogues of the Lie algebra $\mathfrak{g}$ - the current algebra $\mathfrak{g}[t]=\mathfrak{g} \otimes \mathbb{C}[t]$ and the (untwisted) affine Kac-Moody Lie algebra $\widehat{\mathfrak{g}}$ with the natural embedding $\mathfrak{g}[t] \subset \widehat{\mathfrak{g}}$. The $\widehat{\mathfrak{g}}$-analogues of the $\mathfrak{g}$-modules $V_{\lambda}$ are (infinite-dimensional) integrable highest-weight representations $L(\Lambda)$ [Kac]. The central element of $\widehat{\mathfrak{g}}$ acts on $L(\Lambda)$ by a constant called the level of representation. In particular, there are finitely many level 1 integrable modules $L\left(\Lambda_{i}\right), i=0, \ldots, m$, where $L\left(\Lambda_{0}\right)$ is the basic representation. In this paper we will only consider modules $L\left(\ell \Lambda_{i}\right)$ for $\ell \in \mathbb{Z}_{>0}$. The projectivisation $\mathbb{P}\left(L\left(\Lambda_{i}\right)\right)$ contains a partial affine flag variety $\operatorname{Gr}\left(\Lambda_{i}\right)$ as the closure of the orbit of the highest-weight line with respect to the action of the affine Kac-Moody group [Kum2]. The disjoint union $\sqcup_{i=0}^{m} \operatorname{Gr}\left(\Lambda_{i}\right)$ is isomorphic to the affine Grassmannian $\mathrm{Gr}$ for the adjoint Lie group of the Lie algebra $\mathfrak{g}$. 
The Demazure submodules in integrable representations $L(\Lambda)$ are labelled by the elements of the extended affine Weyl group. We will only consider the $\mathfrak{g}[t]$-invariant Demazure modules inside $L\left(\ell \Lambda_{i}\right)$ (note that in general a Demazure module is only acted upon by the Iwahori subalgebra that is strictly contained in the current algebra). In particular, the $\mathfrak{g}[t]$-invariant Demazure modules $D_{1, \lambda}$ inside the level 1 integrable representations are labelled by the dominant integral weights $\lambda$. We denote by $D_{\ell, \lambda}$, $\ell \geq 1$, the level $\ell$ affine Demazure modules contained in the $\ell$ th tensor power of the level 1 module $D_{1, \lambda}$. The projectivised Demazure module $\mathbb{P}\left(D_{1, \lambda}\right)$ contains the spherical Schubert variety $\overline{\mathrm{Gr}}^{\lambda}$ as the orbit closure of the current group action. Thanks to the embedding $\overline{\mathrm{Gr}}^{\lambda} \subset \mathbb{P}\left(D_{1, \lambda}\right)$, the Schubert varieties are equipped with ample line bundle $\mathcal{L}$, such that the dual space of sections of $\mathcal{L}^{\otimes \ell}$ is isomorphic to $D_{\ell, \lambda}$ for any $\ell$. The line bundle $\mathcal{L}$ on a Schubert variety can be also obtained as the restriction of the determinant line bundle on the affine Grassmannian (see [Kum2, Z2]).

The current algebra $\mathfrak{g}[t]$ possesses a remarkable family of cyclic finite-dimensional modules $W_{\lambda}$ called the local Weyl modules (see [CL, CP, FL2, KN, Naoi]). In particular, as a $\mathfrak{g}$-module, $W_{\lambda}$ is isomorphic to the tensor product of fundamental local Weyl modules, where the number of factors of the form $W_{\omega}$ is exactly the coefficient of $\omega$ in the decomposition of $\lambda$. We note that in the simply laced case one has an isomorphism $W_{\lambda} \simeq D_{1, \lambda}$. The global Weyl modules $\mathbb{W}_{\lambda}$ are infinite-dimensional cyclic representations of $\mathfrak{g}[t]$ (see [BF1, CFK, CI, FeMa1, Kato1]). One of the most important properties of the global Weyl modules is the existence of free action of the commutative highest-weight algebra $\mathcal{A}_{\lambda}$, commuting with the $\mathfrak{g}[t]$-action. In particular, one obtains a family of (finite-dimensional) $\mathfrak{g}[t]$ modules, labelled by the closed points in $\operatorname{Spec}\left(\mathcal{A}_{\lambda}\right)$, obtained as fibres of $\mathbb{W}_{\lambda}$ with respect to $\mathcal{A}_{\lambda}$; the local Weyl module is the fibre at the origin.

A generalisation of this picture was suggested in [DF]. The authors introduced a family of cyclic (infinite-dimensional) global Demazure modules $\mathbb{D}(\ell, \underline{\lambda})$ - denoted there as $R\left(D_{\ell, \lambda_{1}}, \ldots, D_{\ell, \lambda_{k}}\right)$ corresponding to a collection of dominant integral nonzero weights $\underline{\lambda} \in P_{+}^{k}$ and an integer $\ell>0$; in particular, if all $\lambda_{i}$ are fundamental and $\ell=1$, then one gets back the global Weyl module (this is no longer true in the case that is not simply laced). The global Demazure modules arise naturally in connection with the study of the projective arc spaces (see [Mu1, Mu2, Nash]). The modules $\mathbb{D}(\ell, \underline{\lambda})$ are acted upon by a commutative (highest-weight) algebra $\mathcal{A}(\underline{\lambda})=\mathcal{A}\left(\lambda_{1}, \ldots, \lambda_{k}\right)$ whose action commutes with the $\mathfrak{g}[t]$ action (see [BCES, EGL, KMSV, SV] for examples of similar algebras). The spectrum $\operatorname{Spec}(\mathcal{A}(\underline{\lambda}))$ is the closure of a stratum of the diagonal stratification of a coloured configuration space of the affine line (see section 3.1 for precise definitions). In particular, a closed point $\mathbf{c} \in \mathbb{A}^{k}$ defines the closed point of the same name in $\operatorname{Spec}(\mathcal{A}(\underline{\lambda}))$. For a point $\mathbf{c} \in \operatorname{Spec}(\mathcal{A}(\underline{\lambda}))$, we denote by $\mathbb{D}(\ell, \underline{\lambda}) \mathbf{c}$ the fibre of the global Demazure module at c. Our first theorem is as follows:

\section{Theorem 1.1.}

(a) Assume that $\lambda_{i} \neq 0$ for all $i$. One has an isomorphism of $\mathfrak{g}[t]$-modules

$$
\mathbb{D}(\ell, \underline{\lambda})_{0} \simeq D_{\ell, \lambda_{1}+\cdots+\lambda_{k}} .
$$

(b) Set $\underline{\lambda} \in P_{+}^{k}, \mu \in P_{+}^{l}$. If $\mathbf{c} \in \mathbb{A}^{k}$ and $\mathbf{d} \in \mathbb{A}^{l}$ have no common entries, then the following factorisation property (an isomorphism of $\mathfrak{g}[t]$-modules) holds:

$$
\mathbb{D}(\ell, \underline{\lambda} \sqcup \underline{\mu})_{(\mathbf{c}, \mathbf{d})} \simeq \mathbb{D}(\ell, \underline{\lambda})_{\mathbf{c}} \otimes \mathbb{D}(\ell, \underline{\mu})_{\mathbf{d}} .
$$

(c) The global Demazure module $\mathbb{D}(\ell, \underline{\lambda})$ is free over $\mathcal{A}(\underline{\lambda})$.

(d) The direct sum of $\mathcal{A}(\underline{\lambda})$-dual modules $\bigoplus_{\ell \geq 0} \mathbb{D}(\ell, \underline{\lambda})^{\vee}$ carries a natural structure of $\mathcal{A}(\underline{\lambda})$-algebra.

The properties of the global Demazure modules collected in Theorem 1.1 are parallel to the properties of the Beilinson-Drinfeld spherical Schubert varieties over the affine line. The main goal of this paper is to describe this relation explicitly. More precisely, we 
$\circ$ identify the projective spectrum of the algebra $\bigoplus_{\ell \geq 0} \mathbb{D}(\ell, \underline{\lambda})^{\vee}$ with the partially symmetrised BD spherical Schubert varieties;

- embed symmetrised BD spherical Schubert varieties into the projectivisation of the vector bundle $\mathcal{D}(\ell, \underline{\lambda})$ obtained as the localisation of the (free) $\mathcal{A}(\underline{\lambda})$-module $\mathbb{D}(\ell, \underline{\lambda})$; and

$\circ$ identify the dual sections of the determinant line bundle on symmetrised BD spherical Schubert varieties with global Demazure modules.

Let us state our results in more detail. Recall that the Beilinson-Drinfeld Grassmannians ( $B D$ Grassmannians for short) are global versions of the affine Grassmannians [BD1, BD2, FBZ, Z2] defined over the powers of an algebraic curve $X$; in this paper we consider only the case $X=\mathbb{A}^{1}$ and denote the corresponding BD Grassmannians by $\mathrm{Gr}_{\mathbb{A}^{k}}$ (see, e.g., [BKK, CK, CW, Kam, MVy] for various applications in geometric representation theory). The Grassmannians $\mathrm{Gr}_{\mathbb{A}^{k}}$ are ind-varieties over the configuration space $\mathbb{A}^{k}$, and the ind-structure is provided by the BD spherical Schubert varieties $\overline{\mathrm{Gr}} \lambda$, labelled by $k$-tuples of dominant coweights $\underline{\lambda}=\left(\lambda_{1}, \ldots, \lambda_{k}\right) \in P_{+}^{k}$. A group scheme $\mathbb{G}(k)$ over $\mathbb{A}^{k}-$

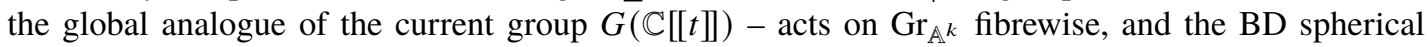
Schubert varieties are the closures of orbits of $\mathbb{G}(k)$ in the generic fibre of $\mathrm{Gr}_{\mathbb{A}^{k}}$; we note that the same group scheme acts on $\mathbb{P}(\mathcal{D}(\ell, \underline{\lambda}))$. The fibres of the projection $\overline{\mathrm{Gr}} \underline{\lambda} \rightarrow \mathbb{A}^{k}$ are products of the spherical Schubert subvarieties of the affine Grassmannian (this is a manifestation of the crucial factorisation property of the BD Grassmannians).

BD Grassmannians carry the ample determinant line bundle $\mathcal{L}$; we keep the same notation for the restriction of this line bundle to the BD spherical Schubert varieties. The space of sections $H^{0}\left(\overline{\mathrm{Gr}}-\underline{\mathcal{L}} \mathcal{L}^{\otimes \ell}\right)$ is naturally a $\mathfrak{g}[t]-\mathbb{C}\left[\mathbb{A}^{k}\right]$-bimodule (we note that the higher cohomology $H^{>0}\left(\overline{\mathrm{Gr}} \underline{\lambda}, \mathcal{L}{ }^{\otimes \ell}\right)$ vanishes). However, as a module over the current algebra, it is not cyclic and hence hard to describe. In order to resolve this problem we consider a partially symmetrised version $\overline{\mathrm{Gr}}(\underline{\lambda})$ of the BD spherical Schubert varieties (see section 3 for a precise definition). The variety $\overline{\mathrm{Gr}^{r}}(\underline{\lambda})$ is equipped with a natural projection onto Spec $(\mathcal{A}(\underline{\lambda}))$, and the determinant line bundle descends to the symmetrised BD spherical Schubert varieties. We prove the following theorem:

Theorem 1.2. For all $\ell \geq 1$ and $\underline{\lambda}=\left(\lambda_{1}, \ldots, \lambda_{k}\right) \in P_{+}^{k}$, such that all $\lambda_{i}$ are nonzero, one has the following:

(a) $a \mathbb{G}(k)$-equivariant embedding

$$
\overline{\mathrm{Gr}}^{(\lambda)} \subset \mathbb{P}(\mathcal{D}(\ell, \underline{\lambda})) ;
$$

(b) an isomorphism of $\operatorname{Spec}(\mathcal{A}(\underline{\lambda}))$-schemes

$$
\overline{\mathrm{Gr}}(\underline{\lambda}) \simeq \operatorname{Proj}\left(\bigoplus_{\ell \geq 0} \mathbb{D}(\ell, \underline{\lambda})^{\vee}\right) ;
$$

(c) an isomorphism of $\mathfrak{g}[t]-\mathcal{A}(\underline{\lambda})$-bimodules

$$
H^{0}\left(\mathrm{Gr}^{(\underline{\lambda})}, \mathcal{L}^{\otimes \ell}\right) \simeq \mathbb{D}(\ell, \underline{\lambda})^{\vee}
$$

(d) an isomorphism of $\mathfrak{g}[t]-\mathbb{C}\left[\mathbb{A}^{k}\right]$-bimodules

$$
H^{0}\left(\mathrm{Gr}^{\underline{\lambda}}, \mathcal{L}^{\otimes \ell}\right) \simeq \mathbb{D}(\ell, \underline{\lambda})^{\vee} \otimes_{\mathcal{A}(\underline{\lambda})} \mathbb{C}\left[\mathbb{A}^{k}\right],
$$

where $M^{\vee}$ stands for the $\mathcal{A}(\underline{\lambda})$-dual to an $\mathcal{A}(\underline{\lambda})$-module $M$.

Let us close with the following remark. In the main body of the paper, we denote the weights and roots of $\mathfrak{g}$ by checked letters (like $\lambda^{\vee}$ and $\alpha^{\vee}$ ) and reserve nonchecked notation for the dual data (coroots and 
coweights). The reason is that the central role in our paper is played by the spherical Schubert varieties in the affine (Beilinson-Drinfeld) Grassmannians. These varieties are naturally labelled by coweights (rather than weights), which explains our choice of notation. Note that in the ADE case all the checks can be removed without any harm. Also, in the simply laced case, if $\lambda_{1}, \ldots, \lambda_{k}$ are all fundamental, and $\lambda=\lambda_{1}+\cdots+\lambda_{k}$, the global Demazure modules $\mathbb{D}(1, \underline{\lambda})$ are nothing but the global Weyl modules $\mathbb{W}_{\lambda}$. However, if $\mathfrak{g}$ is not simply laced, there is no such coincidence anymore. That is why we choose to call $\mathbb{D}(\ell, \underline{\lambda})$ global Demazure modules rather than higher-level global Weyl modules.

Our paper is organised as follows. In section 2 we collect notation and recall main definitions. In section 3 we introduce the symmetrised version of the Beilinson-Drinfeld Grassmannians and Schubert varieties over the spectrum of the highest-weight algebras. In section 4 we study the properties of the global Demazure modules; in particular, we prove that they are free over the highest-weight algebras. In section 5 we compute the spaces of sections of the powers of the determinant line bundle on BD Schubert varieties. In Appendix A, we discuss a connection between global modules and the associativity of the fusion product. We also collect the key objects of the paper in Appendix B.

\section{Generalities}

We start by describing the notation for the key objects of the paper.

\subsection{Classical objects}

Let $\mathfrak{g}$ be a simple Lie algebra over $\mathbb{C}$. The corresponding simply connected (resp., adjoint) complex Lie group will be denoted $G^{\text {sc }}\left(\right.$ resp., $\left.G^{\text {ad }}\right)$. Let $\mathfrak{g}=\mathfrak{n}_{+} \oplus \mathfrak{h} \oplus \mathfrak{n}_{-}$be the Cartan decomposition and let $r=\operatorname{dim} \mathfrak{h}$ be the rank of $\mathfrak{g}$. We denote by $\omega_{1}^{\vee}, \ldots, \omega_{r}^{\vee}$ the fundamental weights of $\mathfrak{g}$ and by $\alpha_{1}^{\vee}, \ldots, \alpha_{r}^{\vee}$ its simple roots. Let $P^{\vee}=\bigoplus_{i=1}^{r} \mathbb{Z} \omega_{i}^{\vee}$ be the weight lattice of $G^{\mathrm{sc}}$ containing the root lattice $Q^{\vee}=\bigoplus_{i=1}^{r} \mathbb{Z} \alpha_{i}^{\vee}$ (which coincides with the weight lattice of $G^{\text {ad }}$ ). Let $P_{+}^{\vee}=\bigoplus_{i=1}^{r} \mathbb{Z}_{\geq 0} \omega_{i}^{\vee} \subset P^{\vee}$ be the set of dominant integral weights. Given $\lambda^{\vee}=\sum_{i=1}^{r} m_{i} \omega_{i}^{\vee} \in P_{+}^{\vee}$, we set $\left|\lambda^{\vee}\right|=\sum_{i=1}^{r} m_{i}$.

For a weight $\lambda^{\vee} \in P_{+}^{\vee}$, let $V_{\lambda^{\vee}}$ be the highest-weight $\lambda^{\vee}$ irreducible $\mathrm{g}$-module; in particular, the highestweight vector of $V_{\lambda^{\vee}}$ is of the $\mathfrak{h}$ weight $\lambda^{\vee}$ and is killed by $\mathfrak{n}_{+}$. Let $W$ be the (finite) Weyl group of $\mathfrak{g}$ with the longest element $w_{0}$. In particular, the lowest-weight vector in $V_{\lambda^{v}}$ is of weight $w_{0} \lambda^{v}$.

We denote by $P=P_{\mathrm{ad}}$ (resp., $Q=P_{\mathrm{sc}}$ ) the coweight lattice of $G^{\text {ad }}$ (resp., of $G^{\mathrm{sc}}$ ). Thus we have perfect pairings $P_{\mathrm{ad}} \times Q^{\vee} \rightarrow \mathbb{Z}, P_{\mathrm{sc}} \times P^{\vee} \rightarrow \mathbb{Z}$. The minimal invariant integral bilinear form on $P_{\mathrm{sc}}$ (such that the square length of a short coroot is 2) gives rise to a linear map $\iota: P_{\mathrm{sc}} \rightarrow Q^{\vee}$. It extends by linearity to the same-named map $P_{\mathrm{sc}} \subset P_{\mathrm{ad}} \stackrel{\iota}{\longrightarrow} Q^{\vee} \otimes_{\mathbb{Z}} \mathbb{Q}$, and $\iota\left(P_{\mathrm{ad}}\right) \subset P^{\vee} \subset Q^{\vee} \otimes_{\mathbb{Z}} \mathbb{Q}$. The resulting map $P=P_{\text {ad }} \rightarrow P^{\vee}$ will be also denoted by $\iota$. In the simply laced case, $\iota: P \rightarrow P^{\vee}$ is an isomorphism.

The fundamental coweights in $P$ are denoted $\omega_{1}, \ldots, \omega_{r}$; the simple coroots in $P$ are denoted by $\alpha_{1}, \ldots \alpha_{r}$. We set $P_{+}=\bigoplus_{i=1}^{r} \mathbb{Z}_{\geq 0} \omega_{i} \subset P$.

\subsection{Current algebra modules}

Let $\mathfrak{g}[t]=\mathfrak{g} \otimes \mathbb{C}[t]$ be the current algebra of $\mathfrak{g}$. In what follows we consider graded $\mathfrak{g}[t]$-modules $M-$ that is, $M=\bigoplus_{i \geq 0} M_{i}, \mathfrak{g} \otimes t^{k}: M_{i} \rightarrow M_{i+k}$. If all $M_{i}$ are finite-dimensional, then the graded character $\operatorname{ch}_{q}(M)$ is a generating function $\sum_{i \geq 0} q^{i} \operatorname{ch} M_{i}$ of the characters of the $\mathfrak{g} \otimes 1$-modules.

A module $M$ is called cyclic if it is generated by a single vector. The cyclic product of two cyclic $\mathfrak{g}[t]$-modules $M_{1}$ and $M_{2}$ with fixed cyclic vectors $w_{1} \in M_{1}$ and $w_{2} \in M_{2}$ is defined as

$$
M_{1} \odot M_{2}=\mathrm{U}(\mathfrak{g}[t]) \cdot w_{1} \otimes w_{2} \subset M_{1} \otimes M_{2} .
$$

For a collection of pairwise distinct complex numbers $c_{1}, \ldots, c_{n}$ and cyclic graded $\mathfrak{g}[t]$-modules $M_{1}, \ldots, M_{n}$, the module $M_{1}\left(c_{1}\right) \otimes \cdots \otimes M_{n}\left(c_{n}\right)$ is known to be cyclic, with the cyclic vector being the tensor product of cyclic vectors of $M_{i}$. Here a $\mathfrak{g}[t]$-module $M_{i}\left(c_{i}\right)$ is defined to be isomorphic 
to $M_{i}$ as a vector space, and the action of the current algebra on it is twisted by the automorphism $x \otimes t^{i} \mapsto x \otimes(t-c)^{i}$. We note that if one starts with graded cyclic modules $M_{i}$, the tensor product $\bigotimes_{i=1}^{n} M_{i}\left(c_{i}\right)$ is not graded in general. The fusion product (graded tensor product) $M_{1} * \cdots * M_{k}$ is defined as the associated graded of $\bigotimes_{i=1}^{n} M_{i}\left(c_{i}\right)$ with respect to the filtration induced by the action of the (t-degree graded) universal enveloping algebra $\mathrm{U}(\mathfrak{g}[t])$ on the tensor product of cyclic vectors of $M_{i}[\mathrm{FeLo}]$.

Let $\mathbb{W}_{\lambda^{\vee}}$ and $W_{\lambda^{\vee}}$ be the global and local Weyl modules of highest weight $\lambda^{\vee}$ over the Lie algebra $\mathfrak{g}[t]$ (see [CP, CL, FL2, Kato1, Naoi]). Let $D_{\lambda}$ be the level 1 affine Demazure module with highest weight $\iota(\lambda)$; in particular, for simply laced algebras $\iota$ is an isomorphism and $W_{\iota}(\lambda) \simeq D_{\lambda}$ for any coweight $\lambda$. For $\lambda \in P_{+}$we denote $\mathbb{W}_{\iota(\lambda)}\left(\right.$ resp., $\left.W_{\iota(\lambda)}\right)$ simply by $\mathbb{W}_{\lambda}$ (resp., $W_{\lambda}$ ).

For $\ell \in \mathbb{Z}_{>0}$ we denote by $D_{\ell, \lambda}$ the level $\ell$ affine Demazure module with highest weight $\ell \iota(\lambda)$ (see section 2.3 for details).

Let $M_{1}, \ldots, M_{k}$ be graded cyclic $\mathfrak{g}[t]$-modules with cyclic vectors $w_{i}$ of dominant nonzero weights such that $t \mathfrak{h}[t]$ annihilates their cyclic vectors. Then we define the global module [DF]

$$
R\left(M_{1}, \ldots, M_{k}\right)=M_{1}[t] \odot \cdots \odot M_{k}[t],
$$

where $M_{i}[t]$ is defined as a module isomorphic to $M_{i} \otimes \mathbb{C}[t]$ as a vector space with the action of $\mathfrak{g}[t]$ given by

$$
x t^{l} . v \otimes t^{k}=\sum_{j=0}^{l}(-1)^{l-j}\left(\begin{array}{l}
l \\
j
\end{array}\right)\left(x t^{j} \cdot v\right) \otimes t^{l+k-j}
$$

for $l, k \in \mathbb{Z}_{\geq 0}, x \in \mathfrak{g}, v \in M_{i}$.

Remark 2.1. The analogous formula used in [DF, FeMa2] has no sign $(-1)^{l-j}$. We introduce it here in order to match the formulas in the Beilinson-Drinfeld setup, where the minus sign pops up via the change of coordinates $t \mapsto t-x$. This sign change obviously produces no harm (simply changing $t \mapsto-t$ in the current algebra parametrisation).

Remark 2.2. The modules $R\left(M_{1}, \ldots, M_{k}\right)$ do depend on the choice of the cyclic vectors $w_{i}$ of $M_{i}$.

The global module $R\left(M_{1}, \ldots, M_{k}\right)$ admits the right action of $\mathrm{U}(\mathfrak{h}[t])$, which commutes with the $\mathfrak{g}[t]$-action. The highest-weight algebra is defined as a quotient of $\mathrm{U}(\mathfrak{h}[t])$ by the annihilator of the cyclic vector $\otimes_{i=1}^{k} w_{i}$ of $R\left(M_{1}, \ldots, M_{k}\right)$. It turns out that the highest-weight algebra depends only on the weights of cyclic vectors $w_{i}$ of $M_{i}$ (not on a particular choice of modules). If the weight of $w_{i}$ is $\iota\left(\lambda_{i}\right)$, then we denote the highest-weight algebra of $R\left(M_{1}, \ldots, M_{k}\right)$ by $\mathcal{A}\left(\lambda_{1}, \ldots, \lambda_{k}\right)$. We will use a shorthand notation $\mathcal{A}(\underline{\lambda})=\mathcal{A}\left(\lambda_{1}, \ldots, \lambda_{k}\right)$, where $\underline{\lambda}=\left(\lambda_{1}, \ldots, \lambda_{k}\right)$.

Since the weight $\iota\left(\lambda_{i}\right)$ subspace of a module $\left.M_{i} \overline{[}\right]$ is isomorphic to a polynomial algebra in one variable, the algebra $\mathcal{A}(\underline{\lambda})$ is naturally embedded into $\bigotimes_{i=1}^{k} \mathcal{A}\left(\lambda_{i}\right) \simeq \mathbb{C}\left[z_{1}, \ldots, z_{k}\right]$. More precisely, $\mathcal{A}(\underline{\lambda})$ is isomorphic to the subalgebra of the polynomial algebra $\mathbb{C}\left[z_{1}, \ldots, z_{k}\right]$ generated by the polynomials

$$
\left\langle\iota\left(\lambda_{1}\right), h\right\rangle z_{1}^{l}+\left\langle\iota\left(\lambda_{2}\right), h\right\rangle z_{2}^{l}+\cdots+\left\langle\iota\left(\lambda_{k}\right), h\right\rangle z_{k}^{l}, \quad l \geq 1, h \in \mathfrak{h} .
$$

Indeed, for $h \in \mathfrak{h}$ and $l>0$, equation (2.1) gives

$$
h t^{l} . \otimes_{i=1}^{k} w_{i}=\sum_{i=1}^{k} \otimes_{j=1}^{i-1} w_{i} \otimes\left\langle\iota\left(\lambda_{j}\right), h\right\rangle w_{j} t^{l} \otimes_{j=i+1}^{k} w_{j}
$$

In particular, if all $\lambda_{i}$ are fundamental coweights, $m_{i}=\#\left\{j: \lambda_{j}=\omega_{i}\right\}$ and $\lambda=\sum_{j=1}^{k} \lambda_{j}=\sum_{i=1}^{r} m_{i} \omega_{i}$, then

$$
\mathcal{A}(\underline{\lambda}) \cong \mathbb{C}\left[z_{1}, \ldots, z_{k}\right]^{S_{m_{1}} \times \cdots \times S_{m_{r}}}=: \mathcal{A}_{\lambda} .
$$


We note the following:

- $\mathcal{A}\left(\lambda_{1}, \ldots, \lambda_{k}\right) \simeq \mathcal{A}\left(\ell \lambda_{1}, \ldots, \ell \lambda_{k}\right)$ for any $\ell \in \mathbb{N}$.

○ If $\mathfrak{g}$ is simply laced, all weights $\lambda_{1}, \ldots, \lambda_{k}$ are fundamental and $\lambda=\sum_{i=1}^{k} \lambda_{i}$, then

$$
R\left(D_{\lambda_{1}}, \ldots, D_{\lambda_{k}}\right) \simeq \mathbb{W}_{\lambda}
$$

If all the coweights $\lambda_{i}$ are fundamental and they sum up to $\lambda$, then we denote by $\mathbb{D}_{\ell, \lambda}$ (global Demazure module) the module $R\left(D_{\ell, \lambda_{1}}, \ldots, D_{\ell, \lambda_{k}}\right)$. In particular, for simply laced $\mathfrak{g}$ one has $\mathbb{D}_{1, \lambda} \simeq \mathbb{W}_{\lambda}$.

If all the coweights $\lambda_{i}$ are fundamental, then the algebra $\mathcal{A}\left(\lambda_{1}, \ldots, \lambda_{k}\right)$ acts freely on $R\left(D_{\lambda_{1}}, \ldots, D_{\lambda_{k}}\right)$ and the fibre at the origin of the global Demazure module is isomorphic to the fusion product $D_{\lambda_{1}} * \cdots * D_{\lambda_{k}} \simeq D_{\lambda_{1}+\cdots+\lambda_{k}}$. The higher-level analogue still holds with fundamental $\lambda_{i}$ replaced by $\ell \lambda_{i}[\mathrm{DF}]$.

As we will prove in Proposition 4.9, for arbitrary dominant coweights $\lambda_{1}, \ldots, \lambda_{k}$, the module $R\left(D_{\ell, \lambda_{1}}, \ldots, D_{\ell, \lambda_{k}}\right)$ is free over $\mathcal{A}\left(\lambda_{1}, \ldots, \lambda_{k}\right)$. We use the notation

$$
\mathbb{D}(\ell, \underline{\lambda})=R\left(D_{\ell, \lambda_{1}}, \ldots, D_{\ell, \lambda_{k}}\right) \text {. }
$$

Remark 2.3. We note that $\mathbb{D}_{\ell, \lambda}=\mathbb{D}(\ell, \underbrace{\omega_{1}, \ldots, \omega_{1}}_{m_{1}}, \ldots, \underbrace{\omega_{r}, \ldots, \omega_{r}}_{m_{r}})$ for a coweight $\lambda=\sum_{j=1}^{r} m_{j} \omega_{j}$.

In what follows we will need the following $\mathcal{A}(\underline{\lambda})$-analogue of the cyclic power. Namely, let

$$
\underbrace{R\left(M_{1}, \ldots, M_{k}\right) \odot_{\mathcal{A}(\underline{\lambda})} \cdots \odot_{\mathcal{A}(\underline{\lambda})} R\left(M_{1}, \ldots, M_{k}\right)}_{\ell}
$$

be the $\mathrm{U}(\mathfrak{g}[t])$-span of the $\ell$ th tensor power of the cyclic (highest-weight) vector of $R\left(M_{1}, \ldots, M_{k}\right)$ inside the $\ell$ th tensor power over $\mathcal{A}(\underline{\lambda})$ of the module $R\left(M_{1}, \ldots, M_{k}\right)$. We denote this cyclic tensor power by $R\left(M_{1}, \ldots, M_{k}\right)_{\mathcal{A}(\underline{\lambda})}^{\odot \ell}$. This object will be important in Proposition 4.3.

\subsection{Affine Lie algebras and Demazure modules}

The details on the material in this subsection can be found in [Kac, Kum2].

Let $\widehat{\mathfrak{g}}=\mathfrak{g} \otimes \mathbb{C}\left[t, t^{-1}\right] \oplus \mathbb{C} K \oplus \mathbb{C} d$ be the untwisted affine Kac-Moody Lie algebra attached to $\mathfrak{g}$. Here $K$ is central element and $d$ is the (negated) degree operator (i.e., $\left[d, x \otimes t^{i}\right]=-i x \otimes t^{i}$ ). The algebra $\widehat{\mathfrak{g}}$ enjoys the Cartan decomposition $\widehat{\mathfrak{g}}=\mathfrak{n}_{+}^{a} \oplus \mathfrak{h}^{a} \oplus \mathfrak{n}_{-}^{a}$, where $\mathfrak{h}^{a}=\mathfrak{h} \otimes 1 \oplus \mathbb{C} K \oplus \mathbb{C} d$ and $\mathfrak{n}_{+}^{a}=\mathfrak{g} \otimes t \mathbb{C}[t] \oplus \mathfrak{n}_{+} \otimes 1$. We denote by $\mathfrak{b}^{a}=\mathfrak{h}^{a} \oplus \mathfrak{n}_{+}^{a}$ the Iwahori subalgebra.

Let $\Lambda_{0}^{\vee}$ be the level 1 basic integrable weight of $\widehat{\mathfrak{g}}$ (in particular, $\Lambda_{0}^{\vee}(\mathfrak{h} \otimes 1)=0$ ). We also denote by $\Lambda_{i}^{\vee}, i=0, \ldots, m$, the set of all integrable level 1 weights of $\widehat{\mathfrak{g}}$ and by $L\left(\Lambda_{i}^{\vee}\right)$ the corresponding highest-weight $\widehat{\mathfrak{g}}$-modules. In particular, the number $m$ of the level 1 modules is equal to the cardinality of $P / Q \simeq \pi_{1}\left(G^{\text {ad }}\right)$.

Let $\operatorname{Gr}\left(\Lambda_{i}^{\vee}\right) \subset \mathbb{P}\left(L\left(\Lambda_{i}^{\vee}\right)\right), i=0, \ldots, m$, be the partial affine flag varieties corresponding to maximal parabolic subgroups of the affine Kac-Moody group $\widehat{G}^{\mathrm{sc}}-$ that is, $\operatorname{Gr}\left(\Lambda_{i}^{\vee}\right) \simeq \widehat{G}^{\mathrm{sc}} / P_{i}$, where $P_{i}$ is the stabiliser of the highest-weight line in $\mathbb{P}\left(L\left(\Lambda_{i}^{\vee}\right)\right)$. By the very definition, each $\operatorname{Gr}\left(\Lambda_{i}^{\vee}\right)$ is equipped with the very ample line bundle $\mathcal{L}$ - the pullback of $\mathcal{O}(1)$ from $\mathbb{P}\left(L\left(\Lambda_{i}^{\vee}\right)\right)$ - and one has the affine analog of the Borel-Weil theorem

$$
H^{0}\left(\operatorname{Gr}\left(\Lambda_{i}^{\vee}\right), \mathcal{L}^{\otimes \ell}\right)^{*} \simeq L\left(\ell \Lambda_{i}^{\vee}\right), \quad \ell \geq 1
$$

where $L\left(\ell \Lambda_{i}^{\vee}\right)$ is the weight $\ell \Lambda_{i}^{\vee}$ integrable (level $\left.\ell\right) \widehat{\mathfrak{g}}$-module and the superscript asterisk denotes the restricted dual space. 
Remark 2.4. The union $\sqcup_{i=0}^{m} \operatorname{Gr}\left(\Lambda_{i}^{\vee}\right)$ is isomorphic to the affine Grassmannian of $G^{\text {ad }}$ (see later for details).

Let $W^{a}=W \ltimes P$ be the extended affine Weyl group (recall that $P$ is the coweight lattice of $G^{\text {ad }}$ ). Then for any $\lambda \in P_{+}$there exists an element $w_{\lambda} \in W^{a}$ such that the $\mathfrak{h}$-weight of $w_{\lambda} \Lambda_{0}^{\vee}$ is equal to $w_{0} \iota(\lambda)$. Let $\Lambda_{i}^{\vee}$ be the unique integrable level 1 weight such that $w_{\lambda} \Lambda_{0}^{\vee}-\Lambda_{i}^{\vee}$ belongs to the root lattice of $\mathfrak{g}$. Let $u_{w_{0} \iota(\lambda)} \in L\left(\Lambda_{i}^{\vee}\right)$ be a weight $w_{0} \iota(\lambda)$ vector. We define the Demazure module $D_{1, \lambda} \subset L\left(\Lambda_{i}^{\vee}\right)$ as the $\mathrm{U}\left(\mathfrak{b}^{a}\right)$ span of the vector $u_{w_{0} \iota}(\lambda)$. An important property of the Demazure modules $D_{1, \lambda}$ is that they are invariant with respect to the whole current algebra $\mathfrak{g}[t] \supset \mathfrak{b}^{a}$. In particular, $D_{1, \lambda}$ contains the irreducible $\mathfrak{g}$-module $V_{\iota(\lambda)}$ as the $\mathrm{U}\left(\mathfrak{n}_{+}\right)$span of $u_{w_{0} \iota(\lambda)}$.

The level $\ell$ Demazure module $D_{\ell, \lambda}$ is defined as the $\mathrm{U}\left(\mathfrak{b}^{a}\right)$ span of the vector $u_{w_{0} \iota(\lambda)}^{\otimes \ell}$. By definition, $D_{\ell, \lambda}$ is a subspace of $L\left(\Lambda_{i}^{\vee}\right)^{\otimes \ell}$. However, it is easy to see that

$$
D_{\ell, \lambda} \subset L\left(\ell \Lambda_{i}^{\vee}\right) \subset L\left(\Lambda_{i}^{\vee}\right)^{\otimes \ell} .
$$

By definition, one gets a natural structure of algebra on the space $D_{\bullet, \lambda}^{*}=\bigoplus_{\ell \geq 0} D_{\ell, \lambda}^{*}$ generated by the degree 1 component $D_{1, \lambda}^{*}$ (we set $D_{0, \lambda}=\mathbb{C}$ ).

We define a spherical Schubert variety $\overline{\mathrm{Gr}}^{\lambda}$ as the closure of the $G^{\mathrm{sc}}(\mathcal{O})$-orbit of the line containing the lowest-weight vector $u_{w_{0} \iota(\lambda)}$ (here $\mathcal{O}=\mathbb{C}[[t]]$ ). Then $\overline{\mathrm{Gr}}^{\lambda}$ is embedded as a closed subscheme into the projectivisation $\mathbb{P}\left(D_{1, \lambda}\right)$ of the Demazure module $D_{1, \lambda}$ (see, e.g., [Ma, Chapter X, Théorème 2. $\Sigma$ ] or [Kum1]). Moreover, $\overline{\mathrm{Gr}}^{\lambda}$ is also embedded as a closed subscheme into the projectivisation of an arbitrary-level Demazure module $D_{\ell, \lambda}$ as the closure of the lowest-weight line.

Remark 2.5. Let $t^{\lambda} \in \overline{\mathrm{Gr}} \lambda \subset \mathbb{P}\left(D_{1, \lambda}\right)$ be the point corresponding to the weight $\iota(\lambda)$ line. Then $\overline{\mathrm{Gr}}^{\lambda}$ is the closure of the $G^{\mathrm{sc}}(\mathcal{O})$-orbit of $t^{\lambda}$.

The embedding $\overline{\mathrm{Gr}}^{\lambda} \subset \mathbb{P}\left(D_{1, \lambda}\right)$ endows $\overline{\mathrm{Gr}}^{\lambda}$ with a very ample line bundle $\mathcal{L}$, the pullback of $\mathcal{O}(1)$. The line bundle $\mathcal{L}$ is a generator of the Picard group of $\overline{\mathrm{Gr}}^{\lambda}$, and one has the isomorphism of $\mathfrak{g}[t]$-modules

$$
H^{0}\left(\overline{\mathrm{Gr}}^{\lambda}, \mathcal{L}^{\otimes \ell}\right)^{*} \simeq D_{\ell, \lambda} \text { for all } \ell \geq 1 .
$$

We obtain a presentation of $\overline{\mathrm{Gr}}^{\lambda}$ as the projective spectrum of the algebra of dual Demazure modules that is, $\overline{\mathrm{Gr}}^{\lambda} \simeq \operatorname{Proj}\left(\bigoplus_{\ell \geq 0} D_{\ell, \lambda}^{*}\right)$.

We have

$$
\operatorname{Gr}\left(\Lambda_{i}^{\vee}\right)=\bigcup_{\lambda: \Lambda_{i}^{\vee}-\iota(\lambda) \in Q^{\vee}+\Lambda_{0}^{\vee}} \overline{\mathrm{Gr}}^{\lambda} .
$$

Also, $\overline{\mathrm{Gr}}^{\lambda} \subset \overline{\mathrm{Gr}}^{\mu}$ if and only if $\mu-\lambda \in \bigoplus_{j=1}^{r} \mathbb{Z}_{\geq 0} \alpha_{j}$.

\subsection{Affine Grassmannians}

The affine Grassmannian of $G^{\text {ad }}$ is $\mathrm{Gr}:=\operatorname{Gr}_{G^{\text {ad }}}=G^{\text {ad }}(\mathcal{K}) / G^{\text {ad }}(\mathcal{O})$, where $\mathcal{K}=\mathbb{C}((t))$ is the Laurentseries ring and $\mathcal{O}=\mathbb{C}[[t]]$ is the Taylor-series ring. The following properties of $\mathrm{Gr}$ can be found in [Z1, Z2, Kum2]:

- The connected components of Gr are in bijection with $P / Q$ - that is, $\pi_{0}(\mathrm{Gr}) \simeq \pi_{1}\left(G^{\mathrm{ad}}\right)$.

○ $\mathrm{Gr}=\sqcup_{i=0}^{m} \mathrm{Gr}\left(\Lambda_{i}^{\vee}\right)$.

○ For any $i=0, \ldots, m$, Pic $\left(\operatorname{Gr}\left(\Lambda_{i}^{\vee}\right)\right)$ is generated by the class of the ample determinant line bundle $\mathcal{L}$.

Recall (see, e.g., [BL]) that the affine Grassmannian Gr is the moduli space of pairs $(\mathcal{P}, \beta)$, where

$\mathcal{P}$ is a $G^{\text {ad }}$-torsor on $\mathbb{A}^{1}$ and $\beta: \mathcal{P}_{\mathbb{A}^{1} \backslash 0} \rightarrow G \times\left(\mathbb{A}^{1} \backslash 0\right)$ is a trivialisation on $\mathbb{A}^{1} \backslash 0$. 
Replacing the point 0 with an arbitrary $c \in \mathbb{A}^{1}$, one gets a version $\mathrm{Gr}_{c}$ of the affine Grassmannian. Clearly, the isomorphism $\mathcal{O} \simeq \mathcal{O}_{c}=\mathbb{C}\left[[t-c]\right.$ induces the isomorphism $\operatorname{Gr}_{c} \simeq$ Gr for any $c$. The schemes $\mathrm{Gr}_{c}$ glue together to the (trivial) bundle $\mathrm{Gr}_{\mathbb{A}^{1}}$ over the affine line.

Remark 2.6. $\mathrm{Gr}_{\mathbb{A}^{1}}$ is the simplest example of a Beilinson-Drinfeld Grassmannian; the general case is discussed in the next section.

\section{Beilinson-Drinfeld Schubert varieties}

We will need several versions of the Beilinson-Drinfeld Schubert varieties [Z1, Z2]. Let us stress from the very beginning that the Beilinson-Drinfeld Grassmannians are defined over (a power of) a smooth curve $X$, but in this paper we consider only the case $X=\mathbb{A}^{1}$. The standard Beilinson-Drinfeld definition produces schemes over affine spaces. We will also need the symmetrised versions with the natural projections to the spectrum of the highest-weight algebras. So we first discuss the properties of the highest-weight algebras, and then we introduce the symmetrised Beilinson-Drinfeld Schubert varieties.

\subsection{The highest-weight algebras}

Let $\underline{\lambda}=\left(\lambda_{1}, \ldots, \lambda_{k}\right)$ be a multiset of dominant coweights. Let $\lambda=\sum_{i=1}^{k} \lambda_{i}=\sum_{j=1}^{r} m_{j} \omega_{j}$ and $N=$ $\sum_{j=1}^{r} m_{j}=|\lambda|$. We set

$$
S_{\lambda}=S_{m_{1}} \times \cdots \times S_{m_{r}} .
$$

Recall the algebras $\mathcal{A}\left(\lambda_{1}, \ldots, \lambda_{k}\right)$ and

$$
\mathcal{A}_{\lambda} \simeq \mathcal{A}(\underbrace{\omega_{1}, \ldots, \omega_{1}}_{m_{1}}, \ldots, \underbrace{\omega_{r}, \ldots, \omega_{r}}_{m_{r}}) \simeq \mathbb{C}\left[z_{1}, \ldots, z_{N}\right]^{S_{\lambda}}
$$

from section 2.2.

Lemma 3.1. There exists a natural surjection of algebras $\mathcal{A}_{\lambda} \rightarrow \mathcal{A}(\underline{\lambda})$.

Proof. Note that $\mathcal{A}_{\lambda}=\mathcal{A}\left(\omega_{a_{1}}, \ldots, \omega_{a_{N}}\right)$, where $\sum_{i=1}^{n} \omega_{a_{i}}=\lambda$. Now it suffices to note that there exists a natural surjection

$$
\mathcal{A}\left(\mu, \lambda_{1}, \ldots, \lambda_{k}\right) \rightarrow \mathcal{A}\left(\mu+\lambda_{1}, \ldots, \lambda_{k}\right)
$$

induced by the surjection of the larger polynomial algebras

$$
\mathbb{C}\left[z_{1}, \ldots, z_{k+1}\right] \rightarrow \mathbb{C}\left[z_{1}, \ldots, z_{k}\right], \quad z_{1} \mapsto z_{1}, \quad z_{2} \mapsto z_{1}, \quad z_{i} \mapsto z_{i-1}, \quad i>2 .
$$

Let $\mathbb{A}^{\lambda}=\mathbb{A}^{N} / S_{\lambda}=\operatorname{Spec} \mathcal{A}_{\lambda}$ be the space of configurations of coloured points on the line $\mathbb{A}^{1}\left(m_{i}\right.$ points of colour $\left.\omega_{i}\right)$. We have the main diagonal $\mathbb{A}^{\lambda} \supset \mathbb{A}^{(\lambda)} \simeq \mathbb{A}^{1}$ formed by all the configurations where all the points coincide. We have a finite morphism of addition of configurations

$$
\operatorname{add}: \mathbb{A}^{v} \times \mathbb{A}^{\mu} \rightarrow \mathbb{A}^{v+\mu}
$$

Iterating it we obtain

$$
\operatorname{add}: \mathbb{A}^{\lambda_{1}} \times \cdots \times \mathbb{A}^{\lambda_{k}} \rightarrow \mathbb{A}^{\lambda}
$$

We define a closed subscheme $\mathbb{A}^{(\lambda)} \subset \mathbb{A}^{\lambda}$ as the add-image of the closed subscheme $\mathbb{A}^{\left(\lambda_{1}\right)} \times \cdots \times \mathbb{A}^{\left(\lambda_{k}\right)} \subset$ $\mathbb{A}^{\lambda_{1}} \times \cdots \times \mathbb{A}^{\lambda_{k}}$ :

$$
\mathbb{A}^{(\lambda)}=\operatorname{add}\left(\mathbb{A}^{\left(\lambda_{1}\right)} \times \cdots \times \mathbb{A}^{\left(\lambda_{k}\right)}\right) \subset \mathbb{A}^{\lambda}
$$


Lemma 3.2. One has

$$
\mathbb{C}[\mathbb{A}(\underline{\lambda})]=\mathcal{A}(\underline{\lambda}) .
$$

Proof. We denote the coordinates on $\mathbb{A}^{N}$ by $x_{i, j}$, where $i=1, \ldots, r$ and $j=1, \ldots, m_{i}$. The group $S_{\lambda}$ acts by permuting the second indices. Let $\lambda_{a}=\sum_{b=1}^{r} m_{a, b} \omega_{b}$ for $a=1, \ldots, k$. In particular, $\sum_{a=1}^{k} m_{a, b}=m_{b}$ for all $b=1, \ldots, r$. Thus the coordinates with a fixed first index are divided into $k$ groups. For each $a=1, \ldots, k$, we combine the corresponding groups for all the possible first indices into one big group $\Gamma_{a}$. Now all the coordinates in $\mathbb{A}^{N}$ are divided into groups $\Gamma_{a}, 1 \leq a \leq k$. We consider the linear subspace $V$ in $\mathbb{A}^{N}$ given by equations $x_{i, j}=x_{i^{\prime}, j^{\prime}}$ whenever $(i, j)$ and $\left(i^{\prime}, j^{\prime}\right)$ lie in the same group $\Gamma_{a}$. We consider the saturation $S_{\lambda} V$ (a union of a few vector subspaces in $\mathbb{A}^{N}$ ). Finally, $\mathbb{A}^{(\lambda)}=\left(S_{\lambda} V\right) / S_{\lambda}$. Now the same argument as in the proof of [BCES, Proposition 2.2] finishes our proof.

Remark 3.3. By construction, $\mathbb{A}^{k}=\mathbb{A}^{\left(\lambda_{1}\right)} \times \cdots \times \mathbb{A}^{\left(\lambda_{k}\right)}$ is finite over $\mathbb{A}(\underline{\lambda})$ (compare $\left.[\mathrm{DF}, \mathrm{BCES}]\right)$. For a closed point $\mathbf{c}=\left(c_{1}, \ldots, c_{k}\right) \in \mathbb{A}^{k}$ we sometimes keep the same notation for its image in $\mathbb{A}(\underline{\lambda})$. For instance, by $\mathbb{C}_{\mathbf{c}}$ we usually mean the one-dimensional $\mathbb{C}[\mathbb{A}(\underline{\lambda})]$-module corresponding to the point $\mathbf{c}$.

\subsection{BD Grassmannians and spherical Schubert varieties}

The Beilinson-Drinfeld Grassmannian $\mathrm{Gr}_{\mathbb{A}^{k}}$ (BD Grassmannian for short) is the moduli space of collections consisting of the points $\left(c_{1}, \ldots, c_{k}\right) \in \mathbb{A}^{k}$, a $G^{\text {ad }}$-torsor $\mathcal{P}$ over $\mathbb{A}^{1}$ and a trivialisation of $\mathcal{P}$ outside the points $c_{i}$.

Example 3.4. If $k=1$, then $\mathrm{Gr}_{\mathbb{A}^{1}}$ is fibred over the affine line with a fibre isomorphic to the affine Grassmannian Gr.

In general, the fibre of the natural projection $\pi: \mathrm{Gr}_{\mathbb{A}^{k}} \rightarrow \mathbb{A}^{k}$ over a point $\left(c_{1}, \ldots, c_{k}\right)$ is isomorphic to the product of $a$ copies of $\mathrm{Gr}$, where $a$ is the number of distinct entries $c_{i}$.

Example 3.5. Let $\AA^{k}, k \geq 2$, be the open subvariety of $\mathbb{A}^{k}$ consisting of points with pairwise distinct coordinates. Then

$$
\pi^{-1}\left(\stackrel{\circ}{\mathbb{A}}^{k}\right) \simeq \stackrel{\circ}{\mathbb{A}}^{k} \times \mathrm{Gr}^{k} .
$$

The BD Grassmannian $\mathrm{Gr}_{\mathbb{A}^{k}}$ enjoys the key factorisation property. We have the addition-ofconfigurations morphism add: $\mathbb{A}^{k} \times \mathbb{A}^{l} \rightarrow \mathbb{A}^{k+l}$ and an open subset $\left(\mathbb{A}^{k} \times \mathbb{A}^{l}\right)_{\text {disj }} \subset \mathbb{A}^{k} \times \mathbb{A}^{l}$ formed by all the pairs of disjoint effective divisors. Then there is a canonical isomorphism

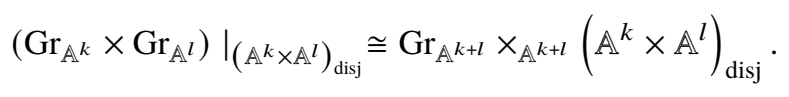

The BD Grassmannian $\mathrm{Gr}_{\mathbb{A}^{k}}$ is an ind-scheme - that is, it is an inductive limit of the finite-dimensional BD Schubert varieties $\overline{\mathrm{Gr}} \underline{\lambda}$ for $k$-tuples of dominant coweights $\underline{\lambda}=\left(\lambda_{1}, \ldots, \lambda_{k}\right)$. More precisely, we consider a group scheme $\mathbb{G}(k)$ over $\mathbb{A}^{k}$, whose fibre over a point $\mathbf{c}=\left(c_{1}, \ldots, c_{k}\right) \in \mathbb{A}^{k}$ is equal to the inverse limit $(n \rightarrow \infty)$

$$
\mathbb{G}(k)_{\mathbf{c}}={\underset{\lim }{n}}_{n} G^{\mathrm{sc}}\left(\mathbb{C}[t] / P(t)^{n}\right), P(t)=\prod_{i=1}^{k}\left(t-c_{i}\right) .
$$

Clearly, a fibre $\mathbb{G}(k)_{\mathbf{c}}$ is isomorphic to the $a$ th power of the group $G^{\mathrm{sc}}(\mathcal{O})$, where $a$ is the number of distinct elements among $c_{i}$. The group $\mathbb{G}(k)$ naturally acts on $\mathrm{Gr}_{\mathbb{A}^{k}}$ fibrewise.

The spherical Schubert varieties in the BD Grassmannian are the closures of the $\mathbb{G}(k)$-orbits in the fibre over the generic point of $\mathbb{A}^{k}$. The orbits are parametrised by the $k$-tuples $\underline{\lambda} \in P_{+}^{k}$. Given such a 
collection, let $t \underline{\lambda}: \mathbb{A}^{k} \rightarrow \mathrm{Gr}_{\mathbb{A}^{k}}$ be a section of $\pi$ such that for $\mathbf{c} \in \AA^{k}$ one has

$$
t \underline{\lambda}(\mathbf{c})=\left(\left(t-c_{1}\right)^{\lambda_{1}}, \ldots,\left(t-c_{k}\right)^{\lambda_{k}}\right) \in \prod_{i=1}^{k} \operatorname{Gr}_{c_{i}},
$$

so the total section is the closure of $t^{\lambda}\left(\AA^{k}\right)$. Now the BD Schubert varieties are defined as the closures of the $\mathbb{G}(k)$-orbits:

$$
\overline{\mathrm{Gr}} \underline{\lambda}=\overline{\mathrm{G}(k) \cdot t \underline{\lambda}} \subset \mathrm{Gr}_{\mathbb{A}^{k}} .
$$

The restriction of $\pi: \mathrm{Gr}_{\mathbb{A}^{k}} \rightarrow \mathbb{A}^{k}$ to $\overline{\mathrm{Gr}} \underline{\lambda}$ is denoted by $\pi_{\lambda}: \overline{\mathrm{Gr}} \underline{\lambda} \rightarrow \mathbb{A}^{k}$. This is a flat morphism, and all the fibres are reduced [Z1, Proposition 1.2.4] (it is proved there for $k=2$, but the proof works for arbitrary $k$ ). The fibre $\overline{\mathrm{Gr}_{\mathbf{c}}} \frac{\lambda}{\lambda}=\pi_{\underline{\lambda}}^{-1}(\mathbf{c})$ over a point $\mathbf{c} \in \mathbb{A}^{k}$ with

$$
c_{1}=\cdots=c_{i_{1}} \neq c_{i_{1}+1}=\cdots=c_{i_{1}+i_{2}} \neq \cdots \neq c_{k-i_{s}+1}=\cdots=c_{k}
$$

is isomorphic to the product

$$
\overline{\mathrm{Gr}}^{\lambda_{1}+\cdots+\lambda_{i_{1}}} \times \overline{\mathrm{Gr}}^{\lambda_{i_{1}+1}+\cdots+\lambda_{i_{1}+i_{2}}} \times \cdots \times \overline{\mathrm{Gr}}^{\lambda_{k-i_{s}+1}+\cdots+\lambda_{k}}
$$

of spherical Schubert varieties in the affine Grassmannian Gr. In particular, the fibre of $\pi_{\underline{\lambda}}$ over the origin (or any other point of the total diagonal) is isomorphic to the spherical Schubert variety $\overline{\mathrm{Gr}}^{\lambda_{1}+\cdots+\lambda_{k}}$.

The BD Grassmannians and the BD Schubert varieties carry the relatively very ample determinant line bundle $\mathcal{L}$. In particular, for any $\ell \geq 1$,

$$
H^{0}\left(\overline{\mathrm{Gr}}_{\mathbf{c}}^{\lambda}, \mathcal{L}_{\mathbf{c}}^{\otimes \ell}\right)^{*} \simeq D_{\ell, \lambda_{1}+\cdots+\lambda_{i_{1}}} \otimes \cdots \otimes D_{\ell, \lambda_{k-i_{S}+1}+\cdots+\lambda_{k}},
$$

where $\mathcal{L}_{\mathbf{c}}$ is the restriction of the line bundle $\mathcal{L}$ to the fibre $\overline{\mathrm{Gr}}_{\mathbf{c}}$.

We also introduce the partially symmetrised (coloured) version $\overline{\mathrm{Gr}}(\underline{\lambda})$ of the BD Schubert varieties. To define it, we first consider the case of fundamental coweights $\lambda_{i}$. So assume that all $\lambda_{i}$ are fundamental that is,

$$
\lambda_{1}=\cdots=\lambda_{m_{1}}=\omega_{1}, \ldots, \lambda_{k-m_{r}+1}=\cdots=\lambda_{k}=\omega_{r}
$$

Let $\lambda=\sum_{i=1}^{k} \lambda_{i}=\sum_{j=1}^{r} m_{j} \omega_{j}$ and $N=|\lambda|=k$. The action of $S_{\lambda}=S_{m_{1}} \times \cdots \times S_{m_{r}}$ on $\mathbb{A}^{N}$ lifts to an action of $S_{\lambda}$ on $\mathrm{Gr}_{\mathbb{A}^{N}}$ such that $\pi_{\lambda}$ is $S_{\lambda}$-equivariant. We define

$$
\operatorname{Gr}_{\mathbb{A}^{\lambda}}:=\operatorname{Gr}_{\mathbb{A}^{N}} / S_{\lambda} .
$$

It is the moduli space of $G^{\text {ad }}$-torsors on $\mathbb{A}^{1}$ trivialised away from an $N$-tuple of points $\left(x_{1}, \ldots, x_{N}\right)$, but we disregard the order within the groups $\left(x_{1}, \ldots, x_{m_{1}}\right), \ldots,\left(x_{N-m_{r}+1}, \ldots, x_{N}\right)$. We introduce the closed subvariety $\overline{\mathrm{Gr}}(\underline{\lambda}) \subset \mathrm{Gr}_{\mathbb{A}^{\lambda}}$ as the categorical quotient

$$
\overline{\mathrm{Gr}}(\underline{\lambda})=\overline{\mathrm{Gr}}-\lambda / S_{\lambda} \subset \mathrm{Gr}_{\mathbb{A}^{N}} / S_{\lambda}=\mathrm{Gr}_{\mathbb{A}^{\lambda}} .
$$

Since the collection $\underline{\lambda}$ of fundamental weights is uniquely determined by their sum $\lambda$, we also use the notation $\overline{\mathrm{Gr}}_{\lambda}$ for $\overline{\mathrm{Gr}}(\bar{\lambda})$.

Now we consider an arbitrary $k$-tuple $\underline{\lambda}=\left(\lambda_{1}, \ldots, \lambda_{k}\right)$, so that $\lambda_{i}$ are not necessarily fundamental coweights. We set again $\lambda=\lambda_{1}+\cdots+\lambda_{k}$. Recall the closed subscheme $\mathbb{A}(\underline{\lambda}) \subset \mathbb{A}^{\lambda}$ introduced in section 3.1. We set

$$
\overline{\mathrm{Gr}}(\underline{\lambda}):=\overline{\mathrm{Gr}}_{\lambda} \times_{\mathbb{A}^{\lambda}} \mathbb{A}(\underline{\lambda}) .
$$


The natural projection $\overline{\mathrm{Gr}}(\underline{\lambda}) \rightarrow \mathbb{A}(\underline{\lambda})$ is denoted $\pi(\underline{\lambda})$.

Note that in case when $(\underline{\lambda})=(\lambda, \lambda, \ldots, \lambda), \mathrm{Gr}^{(\lambda)}$ is a Schubert variety in the symmetrised version of the Beilinson-Drinfeld Grassmannian [Z2].

The determinant line bundle $\mathcal{L}$ descends from $\mathrm{Gr}_{\mathbb{A}^{N}}$ to $\mathrm{Gr}_{\mathbb{A}^{\lambda}}$. We will keep the same notation $\mathcal{L}$ for its restriction to $\overline{\mathrm{Gr}}_{\lambda}$ and to $\overline{\mathrm{Gr}}(\underline{\lambda})$.

\section{Proposition 3.6.}

(a) Let $\lambda_{1}, \ldots, \lambda_{k}$ be fundamental coweights, $\lambda=\sum_{i=1}^{k} \lambda_{i}$. Then one has the base change isomorphism

$$
H^{0}\left(\overline{\mathrm{Gr}}^{\lambda}, \mathcal{L}^{\otimes \ell}\right) \cong H^{0}\left(\overline{\mathrm{Gr}}_{\lambda}, \mathcal{L}^{\otimes \ell}\right) \otimes_{\mathcal{A}_{\lambda}} \mathbb{C}\left[\mathbb{A}^{k}\right]
$$

(b) Let $\lambda_{1}, \ldots, \lambda_{k}$ be arbitrary dominant coweights, $\lambda=\sum_{i=1}^{k} \lambda_{i}$. Then one has the base change isomorphism

$$
H^{0}\left(\overline{\mathrm{Gr}^{\lambda}}, \mathcal{L}^{\otimes \ell}\right) \cong H^{0}\left(\overline{\mathrm{Gr}^{(}}(\underline{\lambda}), \mathcal{L}^{\otimes \ell}\right) \otimes_{\mathcal{A}(\underline{\lambda})} \mathbb{C}\left[\mathbb{A}^{k}\right]
$$

(c) The $\mathbb{C}\left[\mathbb{A}^{k}\right]$-module $H^{0}\left(\overline{\mathrm{Gr}} \underline{-}, \mathcal{L}^{\otimes \ell}\right)$ is free.

(d) The $\mathcal{A}(\underline{\lambda})$-module $H^{0}(\overline{\mathrm{Gr}}(\underline{\lambda}), \mathcal{L} \otimes \ell)$ is free.

Proof. We have a cartesian square

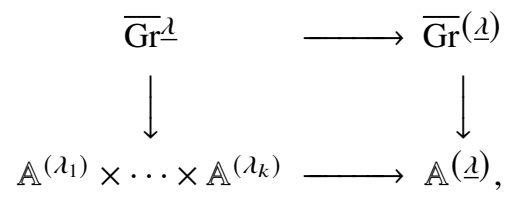

and the determinant line bundle $\mathcal{L}$ on $\overline{\mathrm{Gr}} \underline{\lambda}$ is the pullback of the determinant line bundle $\mathcal{L}$ on $\overline{\mathrm{Gr}}(\underline{\lambda})$. The push-forward of the relatively very ample line bundle $\mathcal{L}^{\otimes \ell}$ from $\overline{\mathrm{Gr}} \lambda$ to $\mathbb{A}^{k}$ is a locally free sheaf $\mathcal{V}$. Indeed, we already know that $\pi_{\lambda}: \overline{\mathrm{Gr}} \stackrel{\lambda}{\rightarrow} \rightarrow \mathbb{A}^{k}$ is flat and all the fibres are reduced. But the dimension of the space of sections of $\mathcal{L}^{\otimes \ell}$ restricted to any fibre is independent of the choice of fibre by [FL1, Theorem 1] or [Z1, Theorem 1.2.2].

Furthermore, the push-forward of $\mathcal{L}^{\otimes \ell}$ from $\overline{\mathrm{Gr}}_{\lambda}$ to $\mathbb{A}^{\lambda}$ is a direct summand of $S_{\lambda}$-invariants in the push-forward of $\mathcal{V}$ from $\mathbb{A}^{k}$ to $\mathbb{A}^{\lambda}$. Hence the push-forward of $\mathcal{L}^{\otimes \ell}$ from $\overline{\mathrm{Gr}}_{\lambda}$ to $\mathbb{A}^{\lambda}$ is a locally free sheaf $\mathcal{W}$ as well. Finally, $\pi_{(\underline{\lambda}) *} \mathcal{L}^{\otimes \ell}$ is the restriction of $\mathcal{W}$ to $\mathbb{A}(\underline{\lambda}) \subset \mathbb{A}^{\lambda}$, and hence $\pi_{(\underline{\lambda}) *} \mathcal{L}^{\otimes \ell}$ is a locally free sheaf on $\mathbb{A}(\underline{\lambda})$ as well. In particular it is flat, and it remains to apply the base change for the cartesian square. This proves (a) and (b).

To prove (c) and (d), note that $H^{0}(\overline{\mathrm{Gr}}(\underline{\lambda}), \mathcal{L} \otimes \ell)$ is projective over $\mathcal{A}(\underline{\lambda})$, because its fibres have the same dimension at every closed point. Since both $\mathcal{A}(\underline{\lambda})$ and $H^{0}\left(\overline{\mathrm{Gr}}(\underline{\lambda}), \mathcal{L}{ }^{\otimes \ell}\right)$ are nonnegatively graded, and the degree 0 part of $\mathcal{A}(\underline{\lambda})$ is $\mathbb{C}$, we conclude by the graded Nakayama lemma that $H^{0}\left(\overline{\mathrm{Gr}}(\underline{\lambda}), \mathcal{L}^{\otimes \ell}\right)$ is free over $\mathcal{A}(\underline{\lambda}){ }^{1}$

\section{Global modules}

In this section, we prove several statements on the global modules defined in [DF]. Although we will be mainly interested in the global Demazure modules, we start in a more general setup.

${ }^{1}$ This last observation is due to Roman Travkin. 
So let $\left\{M_{i}\right\}_{i=1}^{k}$ be cyclic graded $\mathfrak{g}[t]$-modules with cyclic vectors of dominant nonzero weights $\left\{\iota\left(\lambda_{i}\right)\right\}_{i=1}^{k}$, such that $t \mathfrak{h}[t]$ annihilates these cyclic vectors. Recall that it was proved in [DF] that for $\mathbf{c}=\left(c_{1}, \ldots, c_{k}\right)$ lying in some Zariski-open subset of $\mathbb{C}^{k}$, one has

$$
R\left(M_{1}, \ldots, M_{k}\right) \otimes_{\mathcal{A}(\underline{\lambda})} \mathbb{C}_{\mathbf{c}} \simeq \bigotimes_{i=1}^{k} M_{i}\left(c_{i}\right)
$$

(see Remark 3.3).

It was also shown that the fibre of $R\left(M_{1}, \ldots, M_{k}\right)$ at 0 surjects to the fusion product:

$$
R\left(M_{1}, \ldots, M_{k}\right) \otimes_{\mathcal{A}(\underline{\lambda})} \mathbb{C}_{0} \rightarrow M_{1} * \cdots * M_{k} .
$$

In particular, this surjection is an isomorphism if and only if the equality of dimensions $\operatorname{dim}\left(R\left(M_{1}, \ldots, M_{k}\right) \otimes_{\mathcal{A}(\underline{\lambda})} \mathbb{C}_{0}\right)=\prod_{i=1}^{k} \operatorname{dim} M_{i}$ holds.

The next proposition shows that if formula (4.2) is an isomorphism, then the Zariski-open subset for which formula (4.1) holds can be described explicitly:

Proposition 4.1. Suppose an isomorphism $R\left(M_{1}, \ldots, M_{k}\right) \otimes_{\mathcal{A}(\underline{\lambda})} \mathbb{C}_{0} \simeq M_{1} * \cdots * M_{k}$ holds. Then an isomorphism $R\left(M_{1}, \ldots, M_{k}\right) \otimes_{\mathcal{A}(\underline{\lambda})} \mathbb{C}_{\mathbf{c}} \simeq \bigotimes_{i=1}^{k} M_{i}\left(c_{i}\right)$ holds for any $\mathbf{c}$ with pairwise distinct coordinates $c_{i} \neq c_{j}$ (not just for $\mathbf{c}$ in some open subset).

Proof. As explained in [DF], an isomorphism

$$
R\left(M_{1}, \ldots, M_{k}\right) \otimes_{\mathcal{A}(\underline{\lambda})} \mathbb{C}_{0} \simeq M_{1} * \cdots * M_{k}
$$

implies by the semicontinuity theorem the equality

$$
\operatorname{dim}\left(R\left(M_{1}, \ldots, M_{k}\right) \otimes_{\mathcal{A}(\underline{\lambda})} \mathbb{C}_{\mathbf{c}}\right)=\prod_{i=1}^{k} \operatorname{dim} M_{i}
$$

for any c. Hence, it suffices to construct a surjection

$$
R\left(M_{1}, \ldots, M_{k}\right) \otimes_{\mathcal{A}(\underline{\lambda})} \mathbb{C}_{\mathbf{c}} \rightarrow \bigotimes_{i=1}^{k} M_{i}\left(c_{i}\right)
$$

for $\mathbf{c}$ with $c_{i} \neq c_{j}$. For any $i$, clearly one has $M_{i}[t] \rightarrow M_{i}[t] \otimes_{\mathcal{A}\left(\lambda_{i}\right)} \mathbb{C}_{c_{i}} \simeq M_{i}\left(c_{i}\right)$, and hence

$$
R\left(M_{1}, \ldots, M_{k}\right)=\bigodot_{i=1}^{k} M_{i}[t] \rightarrow \bigodot_{i=1}^{k} M_{i}\left(c_{i}\right) \simeq \bigotimes_{i=1}^{k} M_{i}\left(c_{i}\right)
$$

(the last isomorphism is proved in [FeLo, Proposition 1.4]). To show that this surjection factors through $R\left(M_{1}, \ldots, M_{k}\right) \otimes \mathbb{C}_{\mathbf{c}}$, one needs to show that the relations $h t^{n}-\left(\left\langle\iota\left(\lambda_{1}\right), h\right\rangle c_{1}^{n}+\cdots+\left\langle\iota\left(\lambda_{k}\right), h\right\rangle c_{k}^{n}\right)$ hold in the right-hand module for any $h \in \mathfrak{h}$, which is clearly true.

For a global module $R\left(M_{1}, \ldots, M_{k}\right)$, we denote by $R\left(M_{1}, \ldots, M_{k}\right)^{\vee}$ its $\mathcal{A}(\underline{\lambda})$-dual - that is,

$$
R\left(M_{1}, \ldots, M_{k}\right)^{\vee}=\operatorname{Hom}_{\mathcal{A}(\underline{\lambda})}\left(R\left(M_{1}, \ldots, M_{k}\right), \mathcal{A}(\underline{\lambda})\right) .
$$

Remark 4.2. We note that $R\left(M_{1}, \ldots, M_{k}\right)^{\vee}$ carries a natural structure of a $\mathfrak{g}[t]$-module. However, while $R\left(M_{1}, \ldots, M_{k}\right)$ is cyclic, $R\left(M_{1}, \ldots, M_{k}\right)^{\vee}$ does not have to be cyclic or cocyclic. The simplest example pops up for $\mathfrak{g}=\mathfrak{s l}_{2}, k=2$ and $\lambda_{1}=\lambda_{2}=\omega$. We note that if $R\left(M_{1}, \ldots, M_{k}\right)$ is free over $\mathcal{A}(\underline{\lambda})$, then 
the $q$-character of $R\left(M_{1}, \ldots, M_{k}\right)^{\vee}$ is computed as $\left.\operatorname{ch}_{q}\left(R\left(M_{1}, \ldots, M_{k}\right) \otimes_{\mathcal{A}(\underline{\lambda})} \mathbb{C}_{0}\right)\right|_{q \rightarrow q^{-1}} \cdot \operatorname{ch}_{q} \mathcal{A}(\underline{\lambda})$. Hence in our special case one has

$$
\operatorname{ch}_{q} \mathbb{W}_{2 \omega}^{\vee}=q^{-1}+\left(z^{2}+2+z^{-2}\right)+q \cdots,
$$

showing that $\mathbb{W}_{2 \omega}^{\vee}$ is neither cyclic nor cocylic.

Proposition 4.3. Assume that the weights of the cyclic vectors of $\mathfrak{g}[t]$-modules $M_{i}$ are nonzero. Then there is an isomorphism of $\mathfrak{g}[t]-\mathcal{A}(\underline{\lambda})$-bimodules

$$
R\left(M_{1}, \ldots, M_{k}\right)_{\mathcal{A}(\underline{\lambda})}^{\odot \ell} \simeq R\left(M_{1}^{\odot \ell}, \ldots, M_{k}^{\odot \ell}\right)
$$

using the notation of section 2.2.

Proof. Recall that the action of the highest-weight algebra comes from the $\mathrm{U}(\mathfrak{h}[t])$-action. In this proof, we consider global modules with different highest-weight algebras, so we use the notation $\otimes_{\mathrm{U}(\mathfrak{h}[t])}$ instead of $\otimes_{\mathcal{A}(\underline{\lambda})}$, although formally there is no difference.

We first consider an isomorphism

$$
\begin{aligned}
& M_{i}[t] \otimes_{\mathrm{U}(\mathfrak{b}[t])} M_{i}[t] \stackrel{\sim}{\rightarrow}\left(M_{i} \otimes M_{i}\right)[t], \\
& v_{1} t^{k_{1}} \otimes_{\mathrm{U}(\mathfrak{h}[t])} v_{2} t^{k_{2}} \mapsto\left(v_{1} \otimes v_{2}\right) t^{k_{1}+k_{2}}
\end{aligned}
$$

(one can easily check that it is bijective and $\mathfrak{g}[t]$-equivariant). Then we extend it to an isomorphism

$$
\underbrace{\left(\otimes_{i=1}^{k} M_{i}[t]\right) \otimes_{\mathrm{U}(\mathfrak{h}[t])} \cdots \otimes_{\mathrm{U}(\mathfrak{h}[t])}\left(\otimes_{i=1}^{k} M_{i}[t]\right)}_{\ell} \stackrel{\sim}{\rightarrow} \bigotimes_{i=1}^{k} M_{i}^{\otimes \ell}[t] .
$$

Considering the $\mathfrak{g}[t]$-envelopes of the tensor products of cyclic vectors on both sides, we obtain the desired isomorphism

$$
R\left(M_{1}, \ldots, M_{k}\right)_{\mathcal{A}(\underline{\lambda})}^{\odot \ell} \simeq R\left(M_{1}^{\odot \ell}, \ldots, M_{k}^{\odot \ell}\right)
$$

Corollary 4.4. There is a natural structure of a graded $\mathcal{A}(\underline{\lambda})$-algebra on the space

$$
\bigoplus_{\ell \geq 0} R\left(M_{1}^{\odot \ell}, \ldots, M_{k}^{\odot \ell}\right)^{\vee}
$$

where we set $R\left(M_{1}^{\odot 0}, \ldots, M_{k}^{\odot 0}\right)^{\vee}=\mathcal{A}(\underline{\lambda})$.

Proof. The multiplication structure is given by the dual of the map

$$
R\left(M_{1}^{\odot \ell_{1}+\ell_{2}}, \ldots, M_{k}^{\odot \ell_{1}+\ell_{2}}\right) \hookrightarrow R\left(M_{1}^{\odot \ell_{1}}, \ldots, M_{k}^{\odot \ell_{1}}\right) \otimes_{\mathcal{A}(\underline{\lambda})} R\left(M_{1}^{\odot \ell_{2}}, \ldots, M_{k}^{\odot \ell_{2}}\right) .
$$

To make a link to the Beilinson-Drinfeld setup, we consider the global modules with all $M$ s being Demazure modules of the same level $\ell$ and nonzero highest weights. Let us recall the notation

$$
\begin{gathered}
\mathbb{D}(\ell, \underline{\lambda})=R\left(D_{\ell, \lambda_{1}}, \ldots, D_{\ell, \lambda_{k}}\right), \\
\mathbb{D}_{\ell, \lambda}=\mathbb{D}(\ell, \underbrace{\omega_{1}, \ldots, \omega_{1}}_{m_{1}}, \ldots, \underbrace{\omega_{r}, \ldots, \omega_{r}}_{m_{r}}),
\end{gathered}
$$


where $\lambda=\sum_{i=1}^{k} \lambda_{i}=\sum_{j=1}^{r} m_{j} \omega_{j}$. Recall also that $D_{1, \lambda}^{\odot \ell} \simeq D_{\ell, \lambda}$. We get the following version of Corollary 4.4:

Corollary 4.5. There is a natural structure of a graded $\mathcal{A}(\underline{\lambda})$-algebra on the space

$$
\bigoplus_{\ell \geq 0} \mathbb{D}(\ell, \underline{\lambda})^{\vee}
$$

In particular, if all $\lambda_{i}$ are fundamental, we have an $\mathcal{A}_{\lambda \text {-algebra }}$

$$
\mathbb{D}_{\lambda}^{v}=\bigoplus_{\ell \geq 0} \mathbb{D}_{\ell, \lambda}^{\vee}
$$

Remark 4.6. Note that by construction this algebra is generated by its first homogeneous component or in other words, one has a surjection from the symmetric algebra:

$$
\operatorname{Sym}_{\mathcal{A}(\underline{\lambda})}^{\bullet} \mathbb{D}(1, \underline{\lambda})^{\vee} \rightarrow \bigoplus_{\ell \geq 0} \mathbb{D}(\ell, \underline{\lambda})^{\vee}
$$

This means that the $\mathbb{A}^{(\lambda)}$-scheme

$$
\operatorname{Proj}\left(\bigoplus_{\ell \geq 0} \mathbb{D}(\ell, \underline{\lambda})^{\vee}\right)
$$

is a closed subscheme of the projective space

$$
\mathbb{P}_{\mathcal{A}(\underline{\lambda})}(\mathbb{D}(1, \underline{\lambda}))=\operatorname{Proj}\left(\operatorname{Sym}_{\mathcal{A}(\underline{\lambda})}^{\bullet} \mathbb{D}(1, \underline{\lambda})^{\vee}\right)
$$

Remark 4.7. Assume that $\mathfrak{g}$ is simply laced, all $\lambda_{i}$ are fundamental and $\lambda=\sum_{i=1}^{k} \lambda_{i}$. Then $\mathbb{D}(1, \underline{\lambda}) \simeq$ $\mathbb{W}_{\lambda}$ is the global Weyl module. It was proved in [Kato2] that the projective spectrum of the algebra $\bigoplus_{\lambda \in P_{+}} \mathbb{W}_{\lambda}^{*}$ is isomorphic to (the formal version of) the semi-infinite flag variety (see also [Kato1, KNS, BF1, BF2, BF3, FiMi, FeMa1]). There are two important differences between the algebras $\bigoplus_{\lambda \in P_{+}} \mathbb{W}_{\lambda}^{*}$ and $\bigoplus_{\ell \geq 0} \mathbb{D}(\ell, \underline{\lambda})^{\vee}$. First, the sum in the first algebra runs over the dominant integral weights, while in the second case the summation is performed over the nonnegative integers. Second, the dual in the first algebra is taken with respect to the ground field, while in the second algebra one considers the duals with respect to the highest-weight algebra.

Now we will prove that an arbitrary global Demazure module $\mathbb{D}(\ell, \underline{\lambda})$ is free over $\mathcal{A}(\underline{\lambda})$. We start with the simply laced case.

Lemma 4.8. Let $\mathfrak{g}$ be of simply-laced type, $\underline{\lambda}=\left(\lambda_{1}, \ldots, \lambda_{k}\right)$ and $\lambda=\lambda_{1}+\cdots+\lambda_{k}$. Then

$$
\mathbb{D}(\ell, \underline{\lambda}) \otimes_{\mathcal{A}(\underline{\lambda})} \mathbb{C}_{0} \simeq D_{\ell, \lambda}
$$

Proof. Note that this Lemma was already proved in [DF, Proposition 3.2] for the case of all $\lambda_{i}$ fundamental coweights. Thus, fundamental Demazure modules satisfy the condition of Proposition Appendix A.1. Using associativity (Proposition Appendix A.1(c)), we obtain the lemma for arbitrary coweights.

We proceed to an arbitrary type.

\section{Proposition 4.9.}

(a) One has an isomorphism of $\mathfrak{g}[t]$-modules

$$
\mathbb{D}(\ell, \underline{\lambda}) \otimes_{\mathcal{A}(\underline{\lambda})} \mathbb{C}_{0} \simeq D_{\ell, \lambda_{1}+\cdots+\lambda_{k}}
$$


(b) The global Demazure module $\mathbb{D}(\ell, \underline{\lambda})$ is free over $\mathcal{A}(\underline{\lambda})$.

Our proof uses ideas of [FL2, Theorem 8].

Proof. We reduce the general case to the case of $\mathfrak{s l}_{2}$, which is simply laced and hence follows from Lemma 4.8.

As we know (recall formula (4.2)), $\operatorname{dim}\left(\mathbb{D}(\ell, \underline{\lambda}) \otimes_{\mathcal{A}(\underline{\lambda})} \mathbb{C}_{0}\right) \geq \operatorname{dim}\left(D_{\ell, \lambda}\right)$, so it suffices to construct a surjection

$$
\mathbb{D}(\ell, \underline{\lambda}) \otimes_{\mathcal{A}(\underline{\lambda})} \mathbb{C}_{0} \nleftarrow D_{\ell, \lambda}
$$

As was shown in [FL2, J], the defining relations of $D_{\ell, \lambda}$ are

$$
\mathfrak{n}^{+}[t] . v=0, \quad h \cdot v=\ell\langle\iota(\lambda), h\rangle v, \quad t \mathfrak{h}[t] . v=0, \quad\left(f_{\beta} t^{s}\right)^{k_{\beta}+1} \cdot v=0 .
$$

Here $h \in \mathfrak{h}, s \in \mathbb{Z}_{\geq 0}$ and $f_{\beta}$ is the Chevalley generator corresponding to a positive root $\beta^{\vee}$. Finally, $k_{\beta}=\ell \max \left\{0,\left\langle\Lambda_{0}^{\vee}+\iota(\lambda),-\beta+s \frac{(\beta, \beta)}{2} K\right\rangle\right\}$.

The first three relations obviously hold in the left-hand side of formula (4.3), so it remains to show that the last one also does. Consider the $\mathfrak{s l}_{2}$-triple $\mathfrak{s l}_{2}^{\beta}$ corresponding to $\beta^{\vee}$.

It was shown in [FL2, Lemma 7] that there is an $\mathfrak{s l}_{2}^{\beta}[t]$-submodule of $D_{1, \lambda_{i}}$, that is isomorphic to the $\widehat{\mathfrak{s l}_{2}^{\beta}}$ Demazure module $D_{\epsilon, m \omega^{\vee}}$, where $\epsilon=(\beta, \beta) / 2, m=\langle\iota(\lambda), \beta\rangle / \epsilon$ and $\omega^{\vee}$ is the fundamental weight of $\mathfrak{s l}_{2}^{\beta}$. It follows that there is an $\mathfrak{s l}_{2}^{\beta}[t]$-submodule of $D_{\ell, \lambda_{i}}$ that is isomorphic to the $\widehat{\mathfrak{s l}_{2}^{\beta}}$ Demazure module $D_{\ell \epsilon, m \omega^{\vee}}$. Denote it by $M\left(\ell, \iota\left(\lambda_{i}\right)\right)$.

This induces an embedding $M\left(\ell, \iota\left(\lambda_{i}\right)\right)[t] \hookrightarrow D_{\ell, \lambda_{i}}[t]$ and hence, denoting the highest vector of $D_{\ell, \lambda_{i}}$ by $v_{i}$, we have

$$
\begin{aligned}
R\left(M\left(\ell, \iota\left(\lambda_{1}\right)\right), \ldots, M\left(\ell, \iota\left(\lambda_{k}\right)\right)\right) \simeq \mathrm{U}\left(\mathfrak{s l}_{2}^{\beta}[t]\right) \cdot \otimes_{i=1}^{k} v_{i} \hookrightarrow \\
\mathrm{U}(\mathfrak{g}[t]) . \otimes_{i=1}^{k} v_{i} \simeq R\left(D_{\ell, \lambda_{1}}, \ldots D_{\ell, \lambda_{k}}\right) \simeq \mathbb{D}(\ell, \underline{\lambda}) .
\end{aligned}
$$

Thereby, one has a map

$$
R\left(M\left(\ell, \iota\left(\lambda_{1}\right)\right), \ldots, M\left(\ell, \iota\left(\lambda_{k}\right)\right)\right) \otimes_{\mathcal{A}} \mathbb{C}_{0} \rightarrow \mathbb{D}(\ell, \underline{\lambda}) \otimes_{\mathcal{A}(\underline{\lambda})} \mathbb{C}_{0},
$$

where $\mathcal{A}$ is the highest-weight algebra of the global $\mathfrak{s l}_{2}^{\beta}[t]$ Demazure module $R\left(M\left(\ell, \iota\left(\lambda_{1}\right)\right)\right.$, $\left.\ldots, M\left(\ell, \iota\left(\lambda_{k}\right)\right)\right)$ and map (4.4) is induced by the natural inclusion $\mathcal{A} \subset \mathcal{A}(\underline{\lambda})$. Now the left-hand side of map (4.4) is isomorphic to $M(\ell, \iota(\lambda))$, since we are in the simply laced case $\mathfrak{g}=\mathfrak{s l}_{2}^{\beta}$. The required relations $\left(f_{\beta} t^{s}\right)^{k_{\beta}+1} \cdot v=0, s \geq 0$, hold in this module, and hence they hold in $\mathbb{D}(\ell, \underline{\lambda}) \otimes_{\mathcal{A}(\underline{\lambda})} \mathbb{C}_{0}$ as well.

The end of the proof repeats that of Proposition 3.6: $\mathbb{D}(\ell, \underline{\lambda})$ is projective over $\mathcal{A}(\underline{\lambda})$ because its fibres have the same dimension at every closed point. Now since both $\mathbb{D}(\ell, \underline{\lambda})$ and $\mathcal{A}(\underline{\lambda})$ are nonnegatively graded and the degree 0 part of $\mathcal{A}(\underline{\lambda})$ is $\mathbb{C}$, we conclude by the graded Nakayama lemma that $\mathbb{D}(\ell, \underline{\lambda})$ is free over $\mathcal{A}(\underline{\lambda})$.

Now we describe one more relation between global modules that will be used later in the paper:

Proposition 4.10. One has an isomorphism of $\mathfrak{g}[t]$-modules

$$
\mathbb{D}(\ell, \underline{\lambda}) \otimes_{\mathcal{A}(\underline{\lambda})} \bigotimes_{i=1}^{k} \mathcal{A}\left(\ell \lambda_{i}\right) \simeq \mathrm{U}(\mathfrak{g}[t]) \cdot\left(\bigotimes_{i=1}^{k} D_{\ell, \lambda_{i}}[t]_{\ell \iota}\left(\lambda_{i}\right)\right) \subset \bigotimes_{i=1}^{k} D_{\ell, \lambda_{i}}[t],
$$

where $D_{\ell, \lambda_{i}}[t]_{\ell \iota\left(\lambda_{i}\right)}$ denotes the highest-weight part of $D_{\ell, \lambda_{i}}[t]$. 
Note that each algebra $\mathcal{A}\left(\ell \lambda_{i}\right)$ is isomorphic to the algebra of polynomials in one variable. We write $\mathcal{A}\left(\ell \lambda_{i}\right)$ (as opposed to just $\mathbb{C}\left[z_{i}\right]$ ) to point out that these algebras come as the highest-weight spaces of the modules $D_{\ell, \lambda_{i}}[t]$. Further in the paper, in the Beilinson-Drinfeld context we use the notation $\mathbb{C}[\mathbb{A}(\underline{\lambda})] \subset \mathbb{C}\left[\mathbb{A}^{k}\right]$ instead of $\mathcal{A}(\underline{\lambda}) \subset \bigotimes_{i=1}^{k} \mathcal{A}\left(\ell \lambda_{i}\right)$, although it is the same, as explained in section 3.1.

Proof. Let $v$ be the cyclic vector of $\mathbb{D}(\ell, \underline{\lambda})$ and let $v_{i}$ be the cyclic vector of $D_{\ell, \lambda_{i}}[t]$. We define the desired morphism by setting

$$
\begin{aligned}
\phi: \mathbb{D}(\ell, \underline{\lambda}) \otimes_{\mathcal{A}(\underline{\lambda})} \bigotimes_{i=1}^{k} \mathcal{A}\left(\ell \lambda_{i}\right) & \rightarrow \bigotimes_{i=1}^{k} D_{\ell, \lambda_{i}}[t], \\
u . v \otimes_{\mathcal{A}(\underline{\lambda})}\left(h_{1} t^{s_{1}} \otimes \cdots \otimes h_{n} t^{s_{k}}\right) & \mapsto u\left(h_{1} t^{s_{1}} v_{1} \otimes \cdots \otimes h_{n} t^{s_{k}} v_{k}\right),
\end{aligned}
$$

for $u \in \mathrm{U}(\mathfrak{g}[t])$.

It is well defined because $\mathcal{A}\left(\ell \lambda_{i}\right)$ acts on $D_{\ell, \lambda_{i}}[t]$, and hence $\bigotimes_{i=1}^{k} \mathcal{A}\left(\ell \lambda_{i}\right)$ acts on $\bigotimes_{i=1}^{k} D_{\ell, \lambda_{i}}[t]$ commuting with the $\mathfrak{g}[t]$-action.

It remains to prove injectivity. Consider both sides as $\bigotimes_{i=1}^{k} \mathcal{A}\left(\ell \lambda_{i}\right)$-modules. Fibres of both sides at any point $\mathbf{c}=\left(c_{1}, \ldots, c_{k}\right)$ with $c_{i} \neq c_{j}$ are $\bigotimes_{i=1}^{k} D_{\ell, \lambda_{i}}\left(c_{i}\right)$. Therefore $\phi$ is injective on fibres in an open subset, and hence injective.

Example 4.11. Let $\mathfrak{g}=\mathfrak{s l}_{2}, k=2$ and $\lambda_{1}=\lambda_{2}=\omega$. Then one has two embeddings:

$$
\begin{gathered}
\mathbb{W}_{2 \omega} \hookrightarrow \mathbb{W}_{\omega} \otimes \mathbb{W}_{\omega}, \\
\mathbb{W}_{2 \omega} \otimes_{\mathbb{C}\left[z_{1}, z_{2}\right]^{S_{2}}} \mathbb{C}\left[z_{1}, z_{2}\right] \hookrightarrow \mathbb{W}_{\omega} \otimes \mathbb{W}_{\omega} .
\end{gathered}
$$

The image of the first embedding (the special case of Kato's theorem [Kato1, Corollary 3.5]) is the $\mathrm{U}(\mathfrak{g}[t])$-envelope of the tensor product of the highest vectors, while the image of the second embedding is the $\mathrm{U}(\mathfrak{g}[t])$-envelope of the tensor product of the highest-weight components.

Remark 4.12. Due to Proposition 4.9, $\mathbb{D}(\ell, \underline{\lambda})$ is free over $\mathcal{A}(\underline{\lambda})$. Hence, $\mathbb{D}(\ell, \underline{\lambda}) \otimes_{\mathcal{A}(\underline{\lambda})} \bigotimes_{i=1}^{k} \mathcal{A}\left(\ell \lambda_{i}\right)$ is free over $\bigotimes_{i=1}^{k} \mathcal{A}\left(\ell \lambda_{i}\right)$. In particular, this implies

$$
\operatorname{ch}_{q}\left(\mathbb{D}(\ell, \underline{\lambda}) \otimes_{\mathcal{A}(\underline{\lambda})} \bigotimes_{i=1}^{k} \mathcal{A}\left(\ell \lambda_{i}\right)\right)=\operatorname{ch}_{q} D_{\ell, \lambda_{1}+\cdots+\lambda_{k}} \times(1-q)^{-k}
$$

Remark 4.13. In fact, the proof of Proposition 4.10 holds for arbitrary global modules $R\left(M_{1}, \ldots, M_{n}\right)$ without any changes. The isomorphism in the general case is of the form

$$
R\left(M_{1}, \ldots, M_{k}\right) \otimes_{\mathcal{A}(\underline{\lambda})} \bigotimes_{i=1}^{k} \mathcal{A}\left(\lambda_{i}\right) \simeq \mathrm{U}(\mathfrak{g}[t]) \cdot\left(\bigotimes_{i=1}^{k} \mathrm{U}(\mathfrak{h}[t]) v_{i}\right) \subset \bigotimes_{i=1}^{k} R\left(M_{i}\right)
$$

\section{Global Demazure modules and BD Schubert varieties}

\subsection{Sections of the determinant line bundle}

The goal of this section is to identify the global Demazure modules $\mathbb{D}(\ell, \underline{\lambda})$ with the $\mathcal{A}(\underline{\lambda})$-dual of the space of sections $H^{0}\left(\overline{\mathrm{Gr}}(\underline{\lambda}), \mathcal{L}^{\otimes \ell}\right)$. We note that the higher cohomology $H^{>0}\left(\overline{\mathrm{Gr}}(\underline{\lambda}), \mathcal{L}^{\otimes \ell}\right)$ vanishes: as in the proof of Proposition 3.6, it follows from the flatness of $\pi_{(\underline{\lambda})}$ and the fact that the restriction of 
$\mathcal{L}^{\otimes \ell}$ to any fibre of $\pi_{(\underline{\lambda})}$ is very ample. To this end, we first establish an isomorphism

$$
H^{0}\left(\overline{\mathrm{Gr}} \underline{\underline{\lambda}}, \mathcal{L}^{\otimes \ell}\right)^{\vee} \simeq \mathbb{D}(\ell, \underline{\lambda}) \otimes_{\mathcal{A}(\underline{\lambda})} \mathbb{C}\left[\mathbb{A}^{k}\right]
$$

where $\mathbb{A}^{k}=\mathbb{A}^{\left(\lambda_{1}\right)} \times \cdots \times \mathbb{A}^{\left(\lambda_{k}\right)}$ and the notation $M^{\vee}$ stands for the $\mathbb{C}\left[\mathbb{A}^{k}\right]$-dual module to a $\mathbb{C}\left[\mathbb{A}^{k}\right]$ module $M$. In order to compare these two spaces we make the following observation:

Lemma 5.1. There is a homomorphism of Lie groups

$$
G^{\mathrm{sc}}[t] \rightarrow \Gamma\left(\mathbb{A}^{k}, \mathbb{G}(k)\right),
$$

where $\Gamma\left(\mathbb{A}^{k}, \mathbb{G}(k)\right)$ is the group of sections of the group scheme $\mathbb{G}(k)$ over $\mathbb{A}^{k}$.

Proof. Recall that $\mathbb{G}(k)$ is defined as a scheme over $\mathbb{A}^{k}$ whose fibre over a point $\mathbf{c}$ is equal to the inverse limit $(n \rightarrow \infty)$ of the groups $G^{\mathrm{sc}}\left(\mathbb{C}[t] / P(t)^{n}\right)$, where $P(t)=\prod_{i=1}^{k}\left(t-c_{i}\right)$. Now the desired homomorphism is induced by sending the coordinate $t$ in $\mathbb{C}[t]$ to $t\left(\bmod P(t)^{n}\right)$.

Corollary 5.2. The space of sections $H^{0}\left(\overline{\mathrm{Gr}} \stackrel{\lambda}{ }, \mathcal{L}^{\otimes \ell}\right)$ is a $\mathfrak{g}[t]$-module. The $\mathfrak{g}[t]$-action commutes with the natural action of $\mathbb{C}\left[\mathbb{A}^{k}\right]$.

Proof. The first claim is a direct consequence of Lemma 5.1. The second claim is clear because the group scheme $\mathbb{G}(k)$ acts fibrewise.

Now we prove our claim for the case of a single weight.

Lemma 5.3. Let $k=1$ - that is, $\underline{\lambda}=(\lambda), \lambda \in P_{+}$. Then for any $\ell \geq 1$ we have an isomorphism of $\mathfrak{g}[t]$-modules

$$
H^{0}\left(\overline{\mathrm{Gr}}^{(\lambda)}, \mathcal{L}^{\otimes \ell}\right)^{\vee} \simeq D_{\ell, \lambda}[t]
$$

Proof. Recall equation (2.1) for the action of the Lie algebra $\mathfrak{g}[x]$ on $D_{\ell, \lambda}[t] \simeq D_{\ell, \lambda} \otimes \mathbb{C}[t]$ :

$$
\left(g \otimes x^{s}\right)\left(v \otimes t^{a}\right)=\sum_{i=0}^{s}(-1)^{s-i}\left(\begin{array}{l}
s \\
i
\end{array}\right)\left(g \otimes x^{i} \cdot v\right) \otimes t^{a+s-i}, \quad g \in \mathfrak{g} .
$$

Here we deliberately replaced the variable $t$ in $\mathfrak{g}[t]$ with an auxiliary variable $x$ in order to make the picture similar to the BD context.

Now let us identify $x$ with the global coordinate on $\mathbb{A}^{1}$. Then we have an isomorphism of vector spaces

$$
H^{0}\left(\overline{\mathrm{Gr}}^{(\lambda)}, \mathcal{L}^{\otimes \ell}\right)^{\vee} \simeq D_{\ell, \lambda} \otimes \mathbb{C}[x],
$$

where $D_{\ell, \lambda}$ is considered as a $\mathfrak{g}[t]$-module. The action of $\mathfrak{g}[x]$ is induced by the map $x \mapsto x-t$, meaning that the result of the action of $g \otimes x^{s}$ on $v \otimes t^{a}$ is given by the right-hand side of equation (5.1).

Theorem 5.4. Let $\underline{\lambda}=\left(\lambda_{1}, \ldots, \lambda_{k}\right) \in P_{+}^{k}$, all $\lambda_{i}$ nonzero. Then one has an isomorphism of $\mathfrak{g}[t]-\mathbb{C}\left[\mathbb{A}^{k}\right]$ bimodules

$$
H^{0}\left(\overline{\mathrm{Gr}} \underline{\underline{\lambda}}, \mathcal{L}^{\otimes \ell}\right)^{\vee} \simeq \mathbb{D}(\ell, \underline{\lambda}) \otimes_{\mathcal{A}(\underline{\lambda})} \mathbb{C}\left[\mathbb{A}^{k}\right]
$$

where $M^{\vee}$ stands for the $\mathbb{C}\left[\mathbb{A}^{k}\right]$-dual module to a $\mathbb{C}\left[\mathbb{A}^{k}\right]$-module $M$.

Proof. According to Proposition 3.6(c), $H^{0}\left(\overline{\mathrm{Gr}}^{(\lambda)}, \mathcal{L} \otimes \ell\right)^{\vee}$ is free as a $\mathbb{C}\left[\mathbb{A}^{k}\right]$-module. In particular, its $\mathfrak{g}$-highest-weight part $H^{0}\left(\overline{\mathrm{Gr}}-\underline{-}, \mathcal{L}^{\otimes \ell}\right)_{\ell \iota(\lambda)}^{\vee}$ is isomorphic to the free rank 1 module over $\mathbb{C}\left[\mathbb{A}^{k}\right]$. We also 
conclude that

$$
\operatorname{ch}_{q} H^{0}\left(\overline{\mathrm{Gr}}-\underline{\underline{L}}, \mathcal{L}^{\otimes \ell}\right)^{\vee}=\operatorname{ch}_{q} D_{\ell, \lambda} \cdot(1-q)^{-k}
$$

Due to Proposition 4.10 and Lemma 5.3, in order to prove the theorem it is enough to show that

$$
H^{0}\left(\overline{\mathrm{Gr}} \stackrel{\lambda}{ }, \mathcal{L}^{\otimes \ell}\right)^{\vee} \simeq \mathrm{U}(\mathfrak{g}[t]) . \bigotimes_{i=1}^{k} H^{0}\left(\overline{\mathrm{Gr}}^{\left(\lambda_{i}\right)}, \mathcal{L}^{\otimes \ell}\right)_{\ell \iota\left(\lambda_{i}\right)}^{\vee}
$$

where the lower index denotes the corresponding $\mathfrak{g}$-weight subspace.

By Lemma 5.3, $H^{0}\left(\overline{\operatorname{Gr}}^{\left(\lambda_{i}\right)}, \mathcal{L}^{\otimes \ell}\right)_{\ell \iota\left(\lambda_{i}\right)}^{\vee}$ is isomorphic to the polynomial ring in one variable as a vector space. We consider the embedding

$$
H^{0}\left(\overline{\mathrm{Gr}} \underline{\underline{\lambda}}, \mathcal{L}^{\otimes \ell}\right)^{\vee} \hookrightarrow H^{0}\left(\overline{\mathrm{Gr}} \underline{\underline{\lambda}}\left(\AA^{k}\right), \mathcal{L}^{\otimes \ell}\right)^{\vee}
$$

where $\overline{\mathrm{Gr}} \underline{\lambda}\left(\AA^{k}\right) \subset \overline{\mathrm{Gr}} \underline{\underline{\lambda}}$ is $\pi_{\underline{\lambda}}^{-1}\left(\AA^{k}{ }^{k}\right)$, and the embedding (5.4) is induced by the open embedding

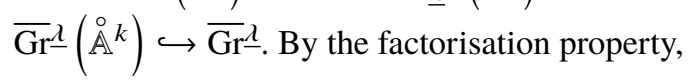

$$
H^{0}\left(\overline{\mathrm{Gr}} \underline{\imath}\left(\AA^{k}\right), \mathcal{L}^{\otimes \ell}\right)^{\vee} \cong \mathbb{C}\left[\AA^{k}\right] \otimes \bigotimes_{i=1}^{k} D\left(\ell, \lambda_{i}\right)
$$

In particular, the highest-weight part $H^{0}\left(\overline{\mathrm{Gr}} \underline{\lambda}\left(\AA^{k}\right), \mathcal{L}^{\otimes \ell}\right)_{\ell \iota(\lambda)}^{\vee}\left(\right.$ with $\left.\lambda=\sum_{i=1}^{k} \lambda_{i}\right)$ is a free rank 1 module over the localisation $\mathbb{C}\left[\AA^{k}\right]$ of the polynomial algebra $\mathbb{C}\left[\mathbb{A}^{k}\right]$.

Thus we have the embeddings

$$
\begin{aligned}
H^{0}\left(\overline{\mathrm{Gr}}^{\underline{\lambda}}, \mathcal{L}^{\otimes \ell}\right)_{\ell \iota(\lambda)}^{\vee} \hookrightarrow H^{0}\left(\overline{\mathrm{Gr}} \underline{\underline{\lambda}}\left(\stackrel{\circ}{\mathbb{A}}^{k}\right), \mathcal{L}^{\otimes \ell}\right)_{\ell \iota(\lambda)}^{\vee} \cong \mathbb{C}\left[\AA^{k}\right] \otimes \bigotimes_{i=1}^{k} D\left(\ell, \lambda_{i}\right)_{\ell \iota(\lambda i)} & \\
& \hookleftarrow \mathbb{C}\left[\mathbb{A}^{k}\right] \otimes_{\mathcal{A}(\underline{\lambda})} \mathbb{D}(\ell, \underline{\lambda})_{\ell \iota(\lambda)}
\end{aligned}
$$

arising from the factorisation property.

We claim that the images of these embeddings coincide. First we consider the case $k=2$. Then the image $I_{\text {left }}$ of the left embedding and the image $I_{\text {right }}$ of the right embedding are both the free rank 1 modules over $\mathbb{C}\left[\mathbb{A}^{2}\right]$ inside the free rank 1 module over $\mathbb{C}\left[\AA^{2}\right]$. If we denote the coordinates in $\mathbb{A}^{2}$ by $z_{1}, z_{2}$, then necessarily $I_{\text {left }}=\left(z_{1}-z_{2}\right)^{a} I_{\text {right }}$ for some $a \in \mathbb{Z}$, and we have to prove $a=0$. Otherwise either $I_{\text {left }} \subsetneq I_{\text {right }}$ (if $a>0$ ) or $I_{\text {right }} \subsetneq I_{\text {left }}$ (if $a<0$ ). In the first case the graded character of $I_{\text {left }}$ is strictly less than the graded character of $I_{\text {right }}$ (termwise), which contradicts the equality $\operatorname{ch}_{q} H^{0}\left(\overline{\mathrm{Gr}}-\underline{\lambda}, \mathcal{L}^{\otimes \ell}\right)^{\vee}=\operatorname{ch}_{q} D_{\ell, \lambda} \cdot(1-q)^{-k}=\operatorname{ch}_{q}\left(\mathbb{C}\left[\mathbb{A}^{k}\right] \otimes_{\mathcal{A}(\underline{\lambda})} \mathbb{D}(\ell, \underline{\lambda})\right)$ by equation (5.2) and Remark 4.12 (in particular, the graded characters of the $\ell \iota(\lambda)$-weight components must coincide as well). The second case similarly leads to a contradiction.

The coincidence of images for general $k$ now follows after localisation at generic points of diagonals in $\mathbb{A}^{k}$ by factorisation. Since we know the coincidence generically and in codimension 1 , it follows everywhere by the algebraic Hartogs lemma: given two locally free sheaves on $\mathbb{A}^{k}$, an isomorphism between them defined off a codimension 2 closed subset of $\mathbb{A}^{k}$ necessarily extends to the whole of $\mathbb{A}^{k}$. 
We conclude the equality

$$
H^{0}\left(\overline{\mathrm{Gr}} \underline{\lambda}, \mathcal{L}^{\otimes \ell}\right)_{\ell \iota(\lambda)}^{\vee}=\mathbb{C}\left[\mathbb{A}^{k}\right] \otimes_{\mathcal{A}(\underline{\lambda})} \mathbb{D}(\ell, \underline{\lambda})_{\ell \iota(\lambda)}
$$

inside $\mathbb{C}\left[\AA^{k}\right] \otimes \bigotimes_{i=1}^{k} D\left(\ell, \lambda_{i}\right)$. But

$$
\begin{aligned}
H^{0}\left(\overline{\mathrm{Gr}}-\underline{\underline{\lambda}}, \mathcal{L}^{\otimes \ell}\right)^{\vee} \supset \mathrm{U}(\mathfrak{g}[t]) H^{0} & \left(\overline{\mathrm{Gr}}-\underline{\underline{L}}, \mathcal{L}^{\otimes \ell}\right)_{\ell \iota(\lambda)}^{\vee} \\
& =\mathrm{U}(\mathfrak{g}[t])\left(\mathbb{C}\left[\mathbb{A}^{k}\right] \otimes_{\mathcal{A}(\underline{\lambda})} \mathbb{D}(\ell, \underline{\lambda})_{\ell \iota(\lambda)}\right)=\mathbb{C}\left[\mathbb{A}^{k}\right] \otimes_{\mathcal{A}(\underline{\lambda})} \mathbb{D}(\ell, \underline{\lambda}) .
\end{aligned}
$$

The equality of characters $\operatorname{ch}_{q} H^{0}\left(\overline{\mathrm{Gr}}-\underline{\mathcal{L}}, \mathcal{L}^{\otimes \ell}\right)^{\vee}=\operatorname{ch}_{q}\left(\mathbb{C}\left[\mathbb{A}^{k}\right] \otimes_{\mathcal{A}(\underline{\lambda})} \mathbb{D}(\ell, \underline{\lambda})\right)$ once again guarantees that the inclusion is actually an equality.

The theorem is proved.

Now to establish a relation between the global Demazure modules and the spaces of sections of determinant line bundles on the symmetrised Schubert varieties, we prove the following theorem:

Theorem 5.5. Set $\lambda_{1}, \ldots, \lambda_{k} \in P_{+}$, all $\lambda_{i}$ nonzero. Then one has an isomorphism of $\mathfrak{g}[t]$-modules

$$
H^{0}\left(\overline{\mathrm{Gr}}(\underline{\lambda}), \mathcal{L}^{\otimes \ell}\right)^{\vee} \simeq \mathbb{D}(\ell, \underline{\lambda})
$$

where $M^{\vee}$ stands for the $\mathcal{A}(\underline{\lambda})$-dual module to an $\mathcal{A}(\underline{\lambda})$-module $M$.

Proof. Using Proposition 3.6 and Theorem 5.4 we get an isomorphism

$$
\mathbb{D}(\ell, \underline{\lambda}) \otimes_{\mathcal{A}(\underline{\lambda})} \mathbb{C}\left[\mathbb{A}^{k}\right] \simeq H^{0}\left(\overline{\mathrm{Gr}}(\underline{\lambda}), \mathcal{L}^{\otimes \ell}\right)^{\vee} \otimes_{\mathcal{A}(\underline{\lambda})} \mathbb{C}\left[\mathbb{A}^{k}\right]
$$

Let $v$ be the cyclic vector of $\mathbb{D}(\ell, \underline{\lambda})$. The vector $v \otimes_{\mathcal{A}(\underline{\lambda})} 1$ on the left-hand side of formula (5.6) is mapped to some vector of the form $w \otimes_{\mathcal{A}(\underline{\lambda})} 1$ on the right-hand side. Using the $\mathfrak{g}[t]$-equivariance we obtain

$$
\mathbb{D}(\ell, \underline{\lambda}) \simeq(\mathrm{U}(\mathfrak{g}[t]) \cdot w) \otimes_{\mathcal{A}(\underline{\lambda})} 1 \subset H^{0}\left(\overline{\mathrm{Gr}}^{(\lambda)}, \mathcal{L}^{\otimes \ell}\right)^{\vee} \otimes_{\mathcal{A}(\underline{\lambda})} 1
$$

Hence there is an embedding $\mathbb{D}(\ell, \underline{\lambda}) \hookrightarrow H^{0}\left(\overline{\mathrm{Gr}}(\underline{\lambda}), \mathcal{L}^{\otimes \ell}\right)^{\vee}$. Using Proposition 4.9, we see that the fibres at 0 of both sides are isomorphic to $D_{\ell, \lambda_{1}+\cdots+\lambda_{k}}$. The graded version of the Nakayama lemma implies that the injective map is a surjection, and thus an isomorphism.

Remark 5.6. If the highest weight of a cyclic $\mathfrak{g}[t]$-module $M$ is 0 , then the module $M[z]$ is not cyclic. That is why we impose the condition $\lambda_{i} \neq 0$ in Theorems 5.4 and 5.5.

It is an easy consequence of these theorems that for the case $\underline{\lambda}=(\mu, 0, \ldots, 0)$ with $\mu_{i} \neq 0$, one has

$$
\begin{aligned}
H^{0}\left(\overline{\mathrm{Gr}}-\underline{\underline{\lambda}}, \mathcal{L}^{\otimes \ell}\right)^{\vee} & \simeq\left(\mathbb{D}(\ell, \underline{\mu}) \otimes_{\mathcal{A}(\underline{\mu})} \mathbb{C}\left[\mathbb{A}^{k}\right]\right) \otimes \mathbb{C}\left[\mathbb{A}^{n}\right], \\
H^{0}\left(\overline{\mathrm{Gr}}(\underline{\lambda}), \mathcal{L}^{\otimes \ell}\right)^{\vee} & \simeq \mathbb{D}(\ell, \underline{\mu}) \otimes \mathbb{C}\left[\mathbb{A}^{(n)}\right] .
\end{aligned}
$$


Corollary 5.7. One has an isomorphism of $\mathbb{A}^{(\underline{\lambda})}$-schemes

$$
\overline{\mathrm{Gr}}(\underline{\lambda}) \simeq \operatorname{Proj}\left(\bigoplus_{\ell \geq 0} \mathbb{D}(\ell, \underline{\lambda})^{\vee}\right) .
$$

Let us consider the special case when all $\lambda_{i}$ are fundamental coweights. In particular, $\mathcal{A}(\underline{\lambda}) \simeq \mathcal{A}_{\lambda}$. We obtain the following corollary:

Corollary 5.8. Assume that all $\lambda_{i}$ are fundamental coweights and let $\lambda=\sum_{i=1}^{k} \lambda_{i}$. Then

(i) $H^{0}\left(\overline{\mathrm{Gr}}(\underline{\lambda}), \mathcal{L}^{\otimes \ell}\right)^{\vee} \simeq \mathbb{D}(\ell, \lambda)$;

(ii) $\overline{\mathrm{Gr}}(\underline{\lambda}) \simeq \operatorname{Proj}\left(\bigoplus_{\ell \geq 0} \mathbb{D}(\ell, \lambda)^{\vee}\right)$.

\subsection{Embeddings of the BD Schubert varieties}

The goal of this section is to show that the global Demazure modules provide projective embeddings of Beilinson-Drinfeld Schubert varieties (generalising a relation between the affine Demazure modules and Schubert varieties).

Thanks to Proposition 4.9, the global Demazure module $\mathbb{D}(\ell, \underline{\lambda})$ is free over $\mathcal{A}(\underline{\lambda})$. Hence one gets a vector bundle $\mathcal{D}(\ell, \underline{\lambda})$ on $\mathbb{A}(\underline{\lambda})=\operatorname{Spec}(\mathcal{A}(\underline{\lambda}))$, whose fibre is given by the fibre of $\mathbb{D}(\ell, \underline{\lambda})$ at a point of the base. We will need the following lemma in order to embed the BD Schubert varieties into the fibrewise projectivised vector bundle $\mathcal{D}(1, \underline{\lambda})$ :

Lemma 5.9. The group scheme $\mathbb{G}(k)$ acts on $\mathcal{D}(\ell, \underline{\lambda})$ fibrewise.

Proof. Recall (see equation (3.2)) that the fibre of $\mathbb{G}(k)$ over a point $\mathbf{c}=\left(c_{1}, \ldots, c_{k}\right) \in \mathbb{A}^{k}$ is equal to the inverse limit

$$
\mathbb{G}(k)_{\mathbf{c}}={\underset{\lim }{m}}_{m} G^{\mathrm{sc}}\left(\mathbb{C}[t] / P(t)^{m}\right), \quad P(t)=\prod_{i=1}^{k}\left(t-c_{i}\right)
$$

We also know that for $\mathbf{c}=(\underbrace{c_{1}, \ldots, c_{1}}_{i_{1}}, \ldots, \underbrace{c_{n}, \ldots, c_{n}}_{i_{n}}) \in \mathbb{C}^{k}$ such that $c_{p} \neq c_{q}$ for $p \neq q$, we have

$$
\mathbb{D}(\ell, \underline{\lambda}) \otimes_{\mathcal{A}(\underline{\lambda})} \mathbb{C}_{\mathbf{c}} \simeq \bigotimes_{p=1}^{n} D_{\ell, \lambda_{i_{1}+\cdots+i_{p-1}}+\cdots+\lambda_{i_{1}+\cdots+i_{p}}}\left(c_{i_{p}}\right)
$$

We conclude that $\mathbb{G}(k)$ acts on $\mathcal{D}(\ell, \underline{\lambda})$ fibrewise.

One has a section $s \underline{\lambda}: \mathbb{A}(\underline{\lambda}) \rightarrow \mathbb{P}(\mathcal{D}(1, \underline{\lambda}))$ of the natural projection map $\mathbb{P}(\mathcal{D}(1, \underline{\lambda})) \rightarrow \mathbb{A}(\underline{\lambda})$ sending a point $\mathbf{c}$ to the highest-weight line of $\mathbb{D}(1, \underline{\lambda}) \otimes_{\mathcal{A}(\underline{\lambda})} \mathbb{C}_{\mathbf{c}}$. By Lemma 5.9, the group of sections of the group scheme $\mathbb{G}(k)$ naturally acts on $\mathbb{P}(\mathcal{D}(1, \underline{\lambda}))$. We obtain the following corollary:

Corollary 5.10. $\overline{\mathrm{Gr}}(\underline{\lambda})$ is equal to the closure of the $\mathbb{G}(k)$-orbit of the section $s^{\lambda}$.

Proof. It follows from definition of $\overline{\mathrm{Gr}} \mathrm{x}$ in section 3 and Theorem 5.5.

\section{Appendix A. On the associativity of the fusion product}

It was conjectured in $[\mathrm{DF}]$ that

$$
R\left(M_{1}, \ldots, M_{k}\right) \otimes_{\mathcal{A}(\underline{\lambda})} \mathbb{C}_{0} \simeq M_{1} * \cdots * M_{k} .
$$


The existence of isomorphism (A.1) implies that the fusion product does not depend on the choice of constants. Now we prove that it also implies the associativity of the fusion product.

Proposition Appendix A.1. Let $N_{1}, \ldots, N_{k}, M_{1}, \ldots, M_{m}$ be finite-dimensional graded cyclic $\mathfrak{g}[t]$ modules with cyclic vectors of weights $\lambda_{1}^{\vee}, \ldots, \lambda_{k}^{\vee}, \mu_{1}^{\vee}, \ldots, \mu_{m}^{\vee}$, such that

$$
R\left(N_{1}, \ldots, N_{k}, M_{1}, \ldots, M_{m}\right) \otimes_{\mathcal{A}\left(\underline{\lambda}^{\vee}, \underline{\mu^{\vee}}\right)} \mathbb{C}_{0} \simeq N_{1} * \cdots * N_{k} * M_{1} * \cdots * M_{m} .
$$

Then the following are true:

(a)

$$
R\left(N_{1}, \ldots, N_{k}\right) \otimes_{\mathcal{A}\left(\underline{\lambda}^{\vee}\right)} \mathbb{C}_{0} \simeq N_{1} * \cdots * N_{k} .
$$

(b)

$$
N_{1} * \cdots * N_{k} * M_{1} * \cdots * M_{m} \simeq\left(N_{1} * \cdots * N_{k}\right) * M_{1} * \cdots * M_{m}
$$

(c)

$$
\begin{aligned}
R\left(R\left(N_{1}, \ldots, N_{k}\right) \otimes_{\mathcal{A}\left(\underline{\lambda}^{\vee}\right)} \mathbb{C}_{0}, M_{1}, \ldots, M_{m}\right) \otimes_{\mathcal{A}\left(\lambda_{1}^{\vee}+\cdots+\lambda_{k}^{\vee}, \underline{\mu}^{\vee}\right)} & \mathbb{C}_{0} \\
& \simeq R\left(N_{1}, \ldots, N_{k}, M_{1}, \ldots, M_{m}\right) \otimes_{\mathcal{A}\left(\underline{\lambda}^{\vee}, \underline{\mu}^{\vee}\right)} \mathbb{C}_{0} .
\end{aligned}
$$

Proof. We consider pairwise distinct $c_{0}, c_{1}, \ldots, c_{m} \in \mathbb{C}$. Let

$$
\mathbf{c}=(\underbrace{c_{0}, \ldots, c_{0}}_{k}, c_{1}, \ldots, c_{m}) \in \mathbb{C}^{k+m} .
$$

Then clearly

$$
\begin{aligned}
& R\left(N_{1}, \ldots, N_{k}, M_{1}, \ldots, M_{m}\right) \otimes_{\mathcal{A}\left(\underline{\lambda}^{\vee}, \underline{\mu}^{\vee}\right)} \mathbb{C}_{\mathbf{c}} \rightarrow \\
& \left(R\left(N_{1}, \ldots, N_{k}\right) \otimes_{\mathcal{A}\left(\underline{\lambda}^{\vee}\right)} \mathbb{C}_{\left(c_{0}, \ldots, c_{0}\right)}\right) \odot\left(R\left(M_{1}, \ldots, M_{m}\right) \otimes_{\mathcal{A}\left(\underline{\mu^{\vee}}\right)} \mathbb{C}_{\left(c_{1}, \ldots, c_{m}\right)}\right) \\
& \simeq\left(R\left(N_{1}, \ldots, N_{k}\right) \otimes_{\mathcal{A}\left(\underline{\lambda}^{\vee}\right)} \mathbb{C}_{0}\right)\left(c_{0}\right) \odot\left(M_{1}\left(c_{1}\right) \otimes \cdots \otimes M_{m}\left(c_{m}\right)\right) \\
& \simeq\left(R\left(N_{1}, \ldots, N_{k}\right) \otimes_{\mathcal{A}\left(\underline{\lambda^{v}}\right)} \mathbb{C}_{0}\right)\left(c_{0}\right) \otimes\left(M_{1}\left(c_{1}\right) \otimes \cdots \otimes M_{m}\left(c_{m}\right)\right) .
\end{aligned}
$$

The last isomorphism holds because of [FeLo, Proposition 1.4].

Because of our assumption on the fibre at 0 of $R\left(N_{1}, \ldots, N_{k}, M_{1}, \ldots, M_{m}\right)$, we conclude that the fibres at all the points have the same dimension, and the surjection (A.2) implies

$$
\begin{aligned}
\prod_{i=1}^{k} \operatorname{dim} N_{i} \times & \prod_{i=1}^{m} \operatorname{dim} M_{i} \geq \operatorname{dim}\left(R\left(N_{1}, \ldots, N_{k}\right) \otimes_{\mathcal{A}\left(\underline{\lambda^{\vee}}\right)} \mathbb{C}_{0}\right) \times \prod_{i=1}^{m} \operatorname{dim} M_{i}, \\
& \prod_{i=1}^{k} \operatorname{dim} N_{i} \geq \operatorname{dim}\left(R\left(N_{1}, \ldots, N_{k}\right) \otimes_{\mathcal{A}\left(\underline{\lambda^{\vee}}\right)} \mathbb{C}_{0}\right) .
\end{aligned}
$$

Comparing with formula (4.2), we obtain part (a) of the proposition. We also conclude that formula (A.2) is an isomorphism. 
Now, as proved in [DF, Proposition 2.11], there is a surjection

$$
\begin{gathered}
N_{1} * \cdots * N_{k} * M_{1} * \cdots * M_{m} \simeq R\left(N_{1}, \ldots, N_{k}, M_{1}, \ldots, M_{m}\right) \otimes_{\mathcal{A}\left(\underline{\underline{\lambda}^{\vee}}, \underline{\mu^{\vee}}\right)} \mathbb{C}_{0} \rightarrow \\
\operatorname{gr}\left(R\left(N_{1}, \ldots, N_{k}, M_{1}, \ldots, M_{m}\right) \otimes_{\mathcal{A}\left(\underline{\lambda^{\vee}}, \underline{\mu^{\vee}}\right)} \mathbb{C}_{\mathbf{c}}\right) \\
\simeq \operatorname{gr}\left(\left(R\left(N_{1}, \ldots, N_{k}\right) \otimes_{\mathcal{A}\left(\underline{\lambda^{\vee}}\right)} \mathbb{C}_{0}\right)\left(c_{0}\right) \otimes\left(M_{1}\left(c_{1}\right) \otimes \cdots \otimes M_{m}\left(c_{m}\right)\right)\right) \\
\simeq\left(N_{1} * \cdots * N_{k}\right) * M_{1} * \cdots * M_{m} .
\end{gathered}
$$

Comparing the dimensions of both sides, we obtain part (b) of the proposition.

To prove part (c), we first prove that

$$
\left(R\left(N_{1}, \ldots, N_{k}\right) \otimes_{\mathcal{A}\left(\underline{\lambda^{\vee}}\right)} \mathbb{C}_{0}\right)[z] \simeq R\left(N_{1}, \ldots, N_{k}\right) \otimes_{\mathcal{A}\left(\underline{\lambda^{\vee}}\right)} \mathcal{A}^{\prime},
$$

where the algebra $\mathcal{A}^{\prime}$ is the algebra of polynomials in one variable, obtained by gluing all the variables in the algebra $\mathcal{A}\left(\underline{\lambda}^{\vee}\right)$ :

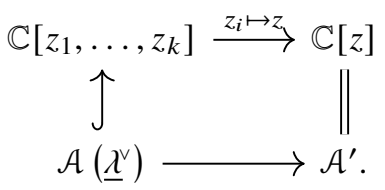

Indeed, it follows from part (a) of the proposition that $R\left(N_{1}, \ldots, N_{k},\right)$ is a free $\mathcal{A}\left(\underline{\lambda}^{\vee}\right)$-module, and hence $R\left(N_{1}, \ldots, N_{k}\right) \otimes_{\mathcal{A}\left(\underline{\lambda}^{\vee}\right)} \mathcal{A}^{\prime}$ is a free $\mathcal{A}^{\prime}$-module. Therefore, we obtain formula (A.3) as an isomorphism of vector spaces. We note that the fibres of the left- and right-hand sides of formula (A.3) at a point $c \in \mathbb{C}$ are isomorphic as $\mathfrak{g}[t]$-modules to $\left(N_{1} * \cdots * N_{k}\right)(c)$. It follows that both sides of formula (A.3) are isomorphic as $\mathfrak{g}[t]$-modules. In particular, there is a surjection

$$
\left(R\left(N_{1}, \ldots, N_{k}\right) \otimes_{\mathcal{A}\left(\underline{\lambda}^{\vee}\right)} \mathbb{C}_{0}\right)[z] \nleftarrow R\left(N_{1}, \ldots, N_{k}\right) .
$$

Using it, we obtain

$$
\begin{gathered}
R\left(R\left(N_{1}, \ldots, N_{k}\right) \otimes_{\mathcal{A}\left(\underline{\lambda}^{\vee}\right)} \mathbb{C}_{0}, M_{1}, \ldots, M_{m}\right) \otimes_{\mathcal{A}\left(\lambda_{1}^{\vee}+\cdots+\lambda_{k}^{\vee}, \underline{\mu^{\vee}}\right)} \mathbb{C}_{0} \\
\simeq\left(\left(R\left(N_{1}, \ldots, N_{k}\right) \otimes_{\mathcal{A}\left(\underline{\lambda^{\vee}}\right)} \mathbb{C}_{0}\right)[z] \odot R\left(M_{1}, \ldots, M_{m}\right)\right) \otimes_{\mathcal{A}\left(\lambda_{1}^{\vee}+\cdots+\lambda_{k}^{\vee}, \underline{\mu^{\vee}}\right)} \mathbb{C}_{0} \\
\nVdash\left(R\left(N_{1}, \ldots, N_{k}\right) \odot R\left(M_{1}, \ldots, M_{m}\right)\right) \otimes_{\mathcal{A}\left(\underline{\lambda}^{\vee}, \underline{\mu}^{\vee}\right)} \mathbb{C}_{0} \\
\simeq R\left(N_{1}, \ldots, N_{k}, M_{1}, \ldots, M_{m}\right) \otimes_{\mathcal{A}\left(\underline{\lambda^{\vee}}, \underline{\underline{v}}\right)} \mathbb{C}_{0} .
\end{gathered}
$$

Comparing the dimensions of the leftmost and rightmost terms, we obtain part (c) of the proposition.

Corollary Appendix A.2 (From the proof of Proposition Appendix A.1). Suppose $M_{1}, \ldots, M_{k}$ are cyclic graded $\mathfrak{g}[t]$ modules such that

$$
R\left(M_{1}, \ldots, M_{k}\right) \otimes_{\mathcal{A}\left(\underline{\lambda}^{\vee}\right)} \mathbb{C}_{0} \simeq M_{1} * \cdots * M_{k} .
$$




$$
\begin{aligned}
& \text { Let } \mathbf{c}=(\underbrace{c_{1}, \ldots, c_{1}}_{i_{1}}, \ldots, \underbrace{c_{n}, \ldots, c_{n}}_{i_{n}}) \in \mathbb{C}^{k} \text { be such that } c_{i} \neq c_{j} \text { for } i \neq j . \text { Then } \\
& R\left(M_{1}, \ldots, M_{k}\right) \otimes_{\mathcal{A}\left(\underline{\lambda}^{\vee}\right)} \mathbb{C}_{\mathbf{c}} \simeq\left(M_{1} * \cdots * M_{i_{1}}\right)\left(c_{1}\right) * \cdots *\left(M_{i_{1}+\cdots+i_{n-1}+1} * \cdots * M_{k}\right)\left(c_{n}\right) .
\end{aligned}
$$

\section{Appendix B. Key objects of the paper}

Simple Lie algebras:

$\mathfrak{g}$ - simple Lie algebra of rank $r$ with Cartan decomposition $\mathfrak{g}=\mathfrak{n}_{+} \oplus \mathfrak{h} \oplus \mathfrak{n}_{-}$

$G^{\text {sc }}$ (resp., $G^{\text {ad }}$ ) - simply connected (resp., adjoint) complex Lie group of $\mathfrak{g}$

$\alpha_{1}^{\vee}, \ldots, \alpha_{r}^{\vee}-$ simple roots

$\omega_{1}^{\vee}, \ldots, \omega_{r}^{\vee}$ - fundamental weights

$\alpha_{1}, \ldots, \alpha_{r}-$ simple coroots

$\omega_{1}, \ldots, \omega_{r}-$ fundamental coweights

$P=\bigoplus_{i=1}^{r} \mathbb{Z} \omega_{i} \supset \bigoplus_{i=1}^{r} \mathbb{Z}_{\geq 0} \omega_{i}-P_{+}$coweight lattice and its dominant cone

$P^{\vee}=\bigoplus_{i=1}^{r} \mathbb{Z} \omega_{i}^{\vee} \supset \bigoplus_{i=1}^{r} \mathbb{Z}_{\geq 0} \omega_{i}^{\vee}-P_{+}^{\vee}$ weight lattice and its dominant cone

$\iota: P \rightarrow P^{\vee}$ - linear map from the coweight lattice to the weight lattice corresponding to the minimal invariant even bilinear form on the coroot lattice ('level 1)

for $\lambda=\sum_{i=1}^{r} m_{i} \omega_{i} \in P_{+}$, we let $|\lambda|=\sum_{i=1}^{r} m_{i}$

$V_{\lambda^{\vee}}$ - irreducible $\mathfrak{g}$-module with highest weight $\lambda^{\vee} \in P_{+}^{\vee}$

Current and affine algebras:

$\mathfrak{g}[t]=\mathfrak{g} \otimes \mathbb{C}[t]-$ current algebra

$W_{\lambda^{\vee}}, \mathbb{W}_{\lambda^{\vee}}\left(\lambda^{\vee} \in P_{+}\right)$- local and global Weyl modules for $\mathfrak{g}[t]$

$S_{\lambda}=\times_{i=1}^{r} S_{m_{i}}-$ symmetric group attached to $\lambda=\sum_{i=1}^{r} m_{i} \omega_{i} \in P_{+}$

$\odot$ - cyclic product

$\widehat{\mathfrak{g}}$ - affine Kac-Moody Lie algebra

$W, W^{a}$ - finite Weyl group and extended affine Weyl group

$D_{\ell, \lambda}\left(\ell \in \mathbb{Z}_{\geq 1}, \lambda \in P_{+}\right)$- level $\ell$ weight $\ell \iota(\lambda)$ affine Demazure module

$\underline{\lambda}=\left(\lambda_{1}, \ldots, \lambda_{k}\right)-$ collection of integral dominant coweights

$\overline{\mathbb{D}}(\ell, \underline{\lambda}) \simeq R\left(D\left(\ell, \lambda_{1}\right), \ldots, D\left(\ell, \lambda_{k}\right)\right)-$ global Demazure module

$\mathbb{D}_{\ell, \lambda} \simeq \mathbb{D}(\ell, \underbrace{\omega_{1}, \ldots, \omega_{1}}_{m_{1}}, \ldots, \underbrace{\omega_{r}, \ldots, \omega_{r}}_{m_{r}})$, where $\lambda=\sum_{i=1}^{r} m_{i} \omega_{i}$

$\mathcal{A}(\underline{\lambda})$ - highest-weight algebra of $\mathbb{D}(\ell, \underline{\lambda})$

$\mathcal{A}_{\lambda} \simeq \mathcal{A}(\underbrace{\omega_{1}, \ldots, \omega_{1}}_{m_{1}}, \ldots, \underbrace{\omega_{r}, \ldots, \omega_{r}}_{m_{r}})-$ highest-weight algebra of $\mathbb{D}_{\ell, \lambda}$

$M^{\vee}=\operatorname{Hom}_{\mathcal{A}(\underline{\lambda})}(M, \mathcal{A}(\underline{\lambda}))-\mathcal{A}(\underline{\lambda})$-dual of an $\mathcal{A}(\underline{\lambda})$-module $M$.

\section{Geometry:}

$\overline{\mathrm{Gr}}^{\lambda} \subset \mathbb{P}(D(1, \lambda))$ - spherical affine Schubert variety

$\mathbb{A}^{\lambda}=\operatorname{Spec}\left(\mathcal{A}_{\lambda}\right)$ - coloured configuration space on the affine line

$\mathbb{A}(\underline{\lambda})=\operatorname{Spec}(\mathcal{A}(\underline{\lambda}))$ - closure of a diagonal stratification stratum in a coloured configuration space on the affine line

$\Lambda_{0}^{\vee}$ - basic level 1 integrable affine weight

$\Lambda_{0}^{\vee}, \Lambda_{1}^{\vee}, \ldots, \Lambda_{m}^{\vee}-$ all level 1 integrable affine weights

$\mathrm{Gr}:=\mathrm{Gr}_{G^{\mathrm{ad}}}=G^{\mathrm{ad}}(\mathbb{C}((t))) / G^{\mathrm{ad}}(\mathbb{C}[[t]])-$ affine Grassmannian of $G^{\mathrm{ad}}$

$\mathrm{Gr} \simeq \sqcup_{i=0}^{m} \operatorname{Gr}\left(\Lambda_{i}^{\vee}\right)-$ decomposition into irreducible components 
$\mathrm{Gr}_{\mathbb{A}^{k}}$ - Beilinson-Drinfeld Grassmannian over $\mathbb{A}^{k} \mathrm{Gr}^{\lambda} \subset \mathrm{Gr}_{\mathbb{A}^{k}}-$ Beilinson-Drinfeld spherical Schubert variety

$\mathrm{Gr}^{(\underline{\lambda})}$ - partially symmetrised Beilinson-Drinfeld spherical Schubert variety over $\mathbb{A}(\underline{\lambda})$

$\mathbb{G}(k)$ - group scheme acting on the Beilinson-Drinfeld Grassmannian

$\mathcal{L}$ - very ample determinant line bundle

$\mathcal{D}(\ell, \underline{\lambda})$ - locally free sheaf on $\mathbb{A}(\underline{\lambda})$, corresponding to the free $\mathcal{A}(\underline{\lambda})$-module $\mathbb{D}(\ell, \underline{\lambda})$

Acknowledgements. The authors would like to thank Syu Kato for his suggestion to study the algebra of dual global Demazure modules. We are indebted to Roman Travkin for his careful reading, corrections and improvements of the first draft. We are also grateful to Alexander Braverman and Boris Feigin for useful discussions. Finally, our thanks go to the anonymous referee for a very careful reading of the manuscript and many useful suggestions. The work was partially supported by grant RSF 19-11-00056. The first author is supported in part by the Simons Foundation.

Conflict of Interest: None.

\section{References}

[BKK] P. Baumann, J. Kamnitzer and A. Knutson, 'The Mirkovic-Vilonen basis and Duistermaat-Heckman measures', Preprint, 2019, arXiv:1905.08460.

[BL] A. Beauville and Y. Laszlo, 'Un lemme de descente', C. R. Math. Acad. Sci. Paris 320(3) (1995), 335-340.

[BD1] A. Beilinson and V. Drinfeld, Chiral Algebras, American Mathematical Society Colloquium Publications vol. 51 (American Mathematical Society, Providence, RI, 2004).

[BD2] A. Beilinson and V. Drinfeld, 'Quantization of Hitchin's integrable system and Hecke eigensheaves', Preprint, 1994, http://www.math.uchicago.edu/mitya/langlands.

[BF1] A. Braverman and M. Finkelberg, 'Weyl modules and $q$-Whittaker functions', Math. Ann. 359(1), (2014), 45-59.

[BF3] A. Braverman and M. Finkelberg, 'Semi-infinite Schubert varieties and quantum K-theory of flag manifolds', $J$. Amer. Math. Soc. 27(4) (2014), 1147-1168.

[BF2] A. Braverman and M. Finkelberg, 'Twisted zastava and $q$-Whittaker functions', J. Lond.Math. Soc. (2) 96(2) (2017), 309-325.

[BCES] A. Brookner, D. Corwin, P. Etingof and S.V. Sam, 'On Cohen-Macaulayness of $S_{n}$-invariant subspace arrangements', Int. Math. Res. Not. IMRN NNN(7) (2016), 2104-2126.

[CK] S. Cautis and J. Kamnitzer, 'Categorical geometric symmetric Howe duality', Selecta Math. (N.S.) 24(2) (2018), 1593-1631.

[CW] S. Cautis and H. Williams, 'Cluster theory of the coherent Satake category', J. Amer. Math. Soc. 32(3) (2019), 709-778.

[CFK] V. Chari, G. Fourier and T. Khandai, 'A categorical approach to Weyl modules', Transform. Groups 15(3) (2010), 517-549.

[CI] V. Chari and B. Ion, 'BGG reciprocity for current algebras', Compos. Math. 151 (2015), 1265-1287.

[CL] V. Chari and S. Loktev, 'Weyl, Demazure and fusion modules for the current algebra of $\mathrm{sl}_{r+1}$ ', Adv. Math. 207(2) (2006), 928-960.

[CP] V. Chari and A. Pressley, 'Weyl modules for classical and quantum affine algebras', Represent. Theory 5 (2001), 191-223.

[DF] I. Dumanski and E. Feigin, 'Reduced arc schemes for Veronese embeddings and global Demazure modules', Preprint, 2019, arXiv:1912.07988.

[EGL] P. Etingof, E. Gorsky and I. Losev, 'Representations of rational Cherednik algebras with minimal support and torus knots', Adv. Math. 277 (2015), 124-180.

[FeLo] B. Feigin and S. Loktev, On Generalized Kostka Polynomials and the Wuantum Verlinde Rule, American Mathematical Society Translations: Series 2 vol. 194 (American Mathematical Society, Providence, RI, 1999).

[FeMa1] E. Feigin and I. Makedonskyi, 'Semi-infinite Plücker relations and Weyl modules', Int. Math. Res. Not. IMRN 2020 14 (2018), 4357-4394.

[FeMa2] E. Feigin and I. Makedonskyi, 'Vertex algebras and coordinate rings of semi-infinite flags', Comm. Math. Phys. 369 (2019), 221-244.

[FiMi] M. Finkelberg and I. Mirkoviić, 'Semi-infinite flags I. Case of global curve $\mathbb{P}^{1}$ ', in Differential Topology, InfiniteDimensional Lie Algebras, and Applications, American Mathematical Society Translations: Series 2 vol. 194 (American Mathematical Society, Providence, RI, 1999), 81-112.

[FL1] G. Fourier and P. Littelmann, 'Tensor product structure of affine Demazure modules and limit constructions', Nagoya Math. J. 182 (2006), 171-198.

[FL2] G. Fourier and P. Littelmann, 'Weyl modules, Demazure modules, KR-modules, crystals, fusion products and limit constructions', Adv. Math. 211(2) (2007), 566-593. 
[FBZ] E. Frenkel and D. Ben-Zvi, Vertex Algebras and Algebraic Curves, second edn, Mathematical Surveys and Monograph vol. 88 (American Mathematical Society, Providence, RI, 2004).

[Fu] W. Fulton, Young Tableaux, with Applications to Representation Theory and Geometry (Cambridge University Press, Cambridge, 1997).

[J] A. Joseph, 'On the Demazure character formula', Ann. Sci. Éc. Norm. Supér. (4) 18(3) (1985), 389-419.

[Kac] V. Kac, Infinite-Dimensional Lie Algebras (Cambridge University Press, Cambridge, UK, 1993).

[Kam] J. Kamnitzer, 'The Beilinson-Drinfeld Grassmannian and symplectic knot homology', in Grassmannians, Moduli Spaces and Vector Bundles, Clay Mathematics Proceedings vol. 14 (American Mathematical Society, Providence, RI, 2011), 81-94.

[KMSV] M. Kasatani, T. Miwa, A.N. Sergeev and A.P. Veselov, 'Coincident root loci and Jack and Macdonald polynomials for special values of the parameters', Contemp. Math. 417 (2006), 207-225.

[Kato1] S. Kato, 'Demazure character formula for semi-infinite flag manifolds', Math. Ann. 371 (2018), no. 3-4, $1769-1801$.

[KNS] S. Kato, S. Naito and D. Sagaki, 'Pieri-Chevalley type formula for equivariant $K$-theory of semi-infinite flag manifolds', Preprint, 2017, arXiv:1702.02408

[KN] R. Kodera and K. Naoi, 'Loewy series of Weyl modules and the Poincaré polynomials of quiver varieties', Publ. Res. Inst. Math. Sci. 48(3) (2012), 477-500.

[Kum1] S. Kumar, 'Demazure character formula in arbitrary Kac-Moody setting', Invent. Math. 89 (1987), $395-423$.

[Kum2] S. Kumar, Kac-Moody Groups, Their Flag Varieties and Representation Theory, Progress in Mathematics vol. 204 (Birkhäuser Boston, Inc., Boston, MA, 2002).

[Ma] O. Mathieu, Formules de caractères pour les algèbres de Kac-Moody générales, Astérisque vol. 159-160 (Société Mathématique de France, Astérisque, 1988), 267 pp.

[MVy] I. Mirković and M. Vybornov, 'Quiver varieties and Beilinson-Drinfeld Grassmannians of type A', Preprint, 2007, arXiv:0712.4160.

[Mu2] M. Mustata, 'Jet schemes of locally complete intersection canonical singularities', Invent. Math. 145 (2001), 397424. With an appendix by D. Eisenbud and E. Frenkel.

[Mu1] M. Mustata, 'Moduli spaces and arcs in algebraic geometry', lecture notes (2006). URL: http://www.math.1sa. umich.edu/mmustata/lectures_arcs.pdf.

[Naoi] K. Naoi, 'Weyl modules, Demazure modules and finite crystals for non-simply laced type', Adv. Math. 229(2) (2012), 875-934.

[Nash] J.F. Nash, 'Arc structure of singularities', Duke Math. J. 81 (1995), 31-38.

[Kato2] S. Kato, 'Frobenius splitting of Schubert varieties of semi-infinite flag manifolds', Preprint, 2018, arXiv:1810. 07106.

[SV] A. Sergeev and A. Veselov, 'Deformed quantum Calogero-Moser systems and Lie superalgebras', Comm. Math. Phys. 245 (2004), 249-278.

[Z1] X. Zhu, 'Affine Demazure modules and $T$-fixed point subschemes in the affine Grassmannian', Adv. Math. 221(2) (2009), 570-600.

[Z2] X. Zhu, 'An introduction to affine Grassmannians and the geometric Satake equivalence', in Geometry of Moduli Spaces and Representation Theory, IAS/Park City Mathematics Series 24 (American Mathematical Society, Providence, RI, 2017) 59-154. 